
\title{
The dopamine transporter gene SLC6A3: multidisease risks
}

\author{
Maarten E. A. Reith ${ }^{1,9}$, Sandhya Kortagere ${ }^{2,9}$, Corinde E. Wiers $\mathbb{D}^{3,4,9}$, Hui Sun ${ }^{3}$, Manju A. Kurian ${ }^{5}$, Aurelio Galli ${ }^{6}$, Nora D. Volkow $\mathbb{D}^{3,7}$ and \\ Zhicheng Lin (D) ${ }^{8}$
}

(c) The Author(s) 2021

The human dopamine transporter gene SLC6A3 has been consistently implicated in several neuropsychiatric diseases but the disease mechanism remains elusive. In this risk synthesis, we have concluded that SLC6A3 represents an increasingly recognized risk with a growing number of familial mutants associated with neuropsychiatric and neurological disorders. At least five loci were related to common and severe diseases including alcohol use disorder (high activity variant), attention-deficit/hyperactivity disorder (low activity variant), autism (familial proteins with mutated networking) and movement disorders (both regulatory variants and familial mutations). Association signals depended on genetic markers used as well as ethnicity examined. Strong haplotype selection and gene-wide epistases support multimarker assessment of functional variations and phenotype associations. Inclusion of its promoter region's functional markers such as DNPi (rs67175440) and 5'VNTR (rs70957367) may help delineate condensate-based risk action, testing a locus-pathway-phenotype hypothesis for one gene-multidisease etiology.

Molecular Psychiatry (2022) 27:1031-1046; https://doi.org/10.1038/s41380-021-01341-5

\section{INTRODUCTION}

Dopamine (DA) plays a crucial role in multiple brain functions [1-4] and is also implicated in circadian rhythms and sleep [5, 6], inflammation $[7,8]$, heart failure $[9,10]$ and cancer [11-13]. Since the plasma membrane human DA transporter protein (hDAT) is one of the principal regulators of synaptic DA transmission [14], genetic variation in the coding gene, $S L C 6 A 3$ in the human chromosome 5 (chr5), may affect SLC6A3 function, alter hDAT's density, DA reuptake activity, and the dynamics of DA neurotransmission, contributing to pathophysiology in both central (CNS) and peripheral nervous systems.

Varying SLC6A3 sequence has been correlated to many environment-sensitive psychiatric diseases such as substance use disorders (SUDs), major depressive disorder (MDD), attention deficit hyperactivity disorder (ADHD) and Parkinson's disease (PD). Observed comorbidity presents tremendous clinical challenges [15]. In vivo activity of Slc6a3 (low case for animal genes) is regulated by environmental risk factors including stimulant drugs [16-18], stressors [19, 20] and high-fat food [21] (increasing its activity), environmental enrichment (decreasing its activity) [22], as well as medications [23-25]. However, these regulations remain mechanistically unclear for humans with the related diseases.

Understanding SLC6A3's functional variants is required to delineate individual variation in DA-related pathophysiology and response to the environment [26, 27]. To enhance our understanding, we performed a risk synthesis. Risk synthesis is not risk analysis, review or meta-analysis alone. Instead, it utilizes available information from various approaches, published and/or newly collected, to clarify disease mechanisms in humans. At least three infectious disease studies have partly applied this format of study at behavioral and/or molecular levels [28-30]. This risk synthesis study utilized functional evidence from eight SLC6A3-focused approaches, including familial hDAT mutants (mostly review) with their molecular modeling (new data), case-control associations (review), recombination hotspots of SLC6A3 (new data), hDAT imaging vs. genotype correlational analyses (new data), postmortem mRNA density (new data), transcriptional assays in vitro (review), phylogenomics \& intragenic epistases (new data), and intrinsically disordered regions (IDRs) of transcription factors (TFs) analysis (new data). The Methods section below will describe the collection of the indicated new data.

\section{METHODS}

By its methodological nature the risk synthesis relies on both data already available in the literature and newly collected information via secondary analysis of previously collected samples or data (for study design, see Fig. 1). This section describes the methods used for such secondary analyses and new data collections. Synthesis of all eight-approaches' information allows proposing a hypothesis for SLC6A3's multidisease mechanism.

\section{Molecular modeling of familial hDAT mutants}

Drosophila melanogaster DAT structure (pdb code 4XP4 [31]) was used to model the wildtype (WT) hDAT and each mutation using homology

\footnotetext{
${ }^{1}$ Department of Psychiatry, New York University School of Medicine, New York City, NY 10016, USA. ${ }^{2}$ Department of Microbiology and Immunology, Drexel University College of Medicine, Philadelphia, PA 19129, USA. ${ }^{3}$ Laboratory of Neuroimaging, National Institute on Alcohol Abuse and Alcoholism, Bethesda, MD 20817, USA. ${ }^{4}$ Department of Psychiatry, Perelman School of Medicine, University of Pennsylvania, Philadelphia, PA 19104, USA. ${ }^{5}$ Molecular Neurosciences, Developmental Neurosciences, Zayed Centre for Research into Rare Diseases in Children, UCL Great Ormond Street Institute of Child Health, and Department of Neurology, Great Ormond Street Hospital, London WC1N 1EH, UK. ${ }^{6}$ Department of Surgery, University of Alabama at Birmingham, Birmingham, AL 35294, USA. ${ }^{7}$ National Institute on Drug Abuse, Bethesda, MD 20817, USA. ${ }^{8}$ Laboratory of Psychiatric Neurogenomics, McLean Hospital, and Department of Psychiatry, Harvard Medical School, Belmont, MA 02478, USA. ${ }^{9}$ These authors contributed equally: Maarten E. A. Reith, Sandhya Kortagere, Corinde E. Wiers. ${ }^{\text {email: }}$ zlin@mclean.harvard.edu
}

Received: 17 May 2021 Revised: 28 September 2021 Accepted: 1 October 2021

Published online: 14 October 2021 


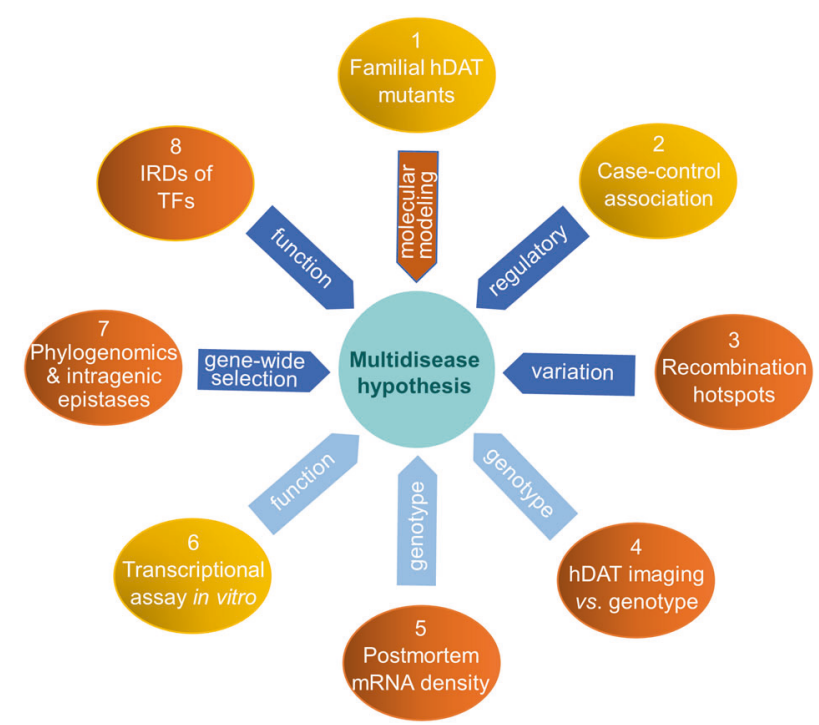

Fig. 1 Study design. Eight channel-synthesis of risk knowledge for functionally polymorphic and vulnerable $S L C 6 A 3$, allowing proposing of a multidisease hypothesis. Mustard oval, reviewed data; orange (1/molecular modeling, 3, 4, 5, 7, 8), new data whose collections are described in the same order in Methods section; blue arrow, mechanism; light blue arrow, functional approach; IDRs of TFs, intrinsically disordered regions of transcription factors.

modeling techniques with Modeler (ver 9.23), followed by structural refining using Molecular Operating Environment, as previously reported [32], and modeling results are detailed by Fig. 2a and associated supplementary text information.

\section{Recombination hotspots}

Polymorphism information in 26 populations (2268 individuals informative for this gene) of the 1000 Genomes Project (1KGP) [33] was used to reveal recombination hotspots in SLC6A3 by using the published FastEPRR protocol [34]. The results are integrated in Fig. $2 b$.

\section{hDAT imaging vs. genotype correlational analysis consisted of the following four steps}

\section{Positron emission tomography (PET)}

Measures of hDAT availability in the human brain had been collected using PET and $\left[{ }^{11} \mathrm{C}\right]$ cocaine, a radioligand to measure hDAT, and retrieved from the imaging dataset of the BNL Brain Imaging Center. All PET scans were performed on a Siemens, HR + scanner in 3D mode via procedures as reported [35] along with the analytical approach to quantify the availability, which was estimated as $B_{\max } / K_{\mathrm{D}}[36,37]$. For this study we only used data obtained in 62 healthy male controls (average age at 35.1 years with a standard deviation of $6.7 ; N=27$ African Americans, 28 Caucasians, 3 Hispanics, and 4 multiracial).

2. Genotyping of DNPi and 5'VNTR

By using REDTaq ReadyMix ${ }^{\text {TM }}$ PCR Reaction Mix from Sigma, DNPi was amplified by using primers $5^{\prime}$-gaatacagatgaacagtcatgaagac- $3^{\prime}$ and $5^{\prime}$-ctcatgggcacactgggagttgagg- $3^{\prime}$, and 40 cycles of $94^{\circ} \mathrm{C}-30 \mathrm{sec}$, $58^{\circ} \mathrm{C}-30 \mathrm{sec}$, and $72^{\circ} \mathrm{C}-45 \mathrm{sec}$, both using $72^{\circ} \mathrm{C}-10 \mathrm{~min}$ as the final PCR extension. DNPi product was subject to allelic BseRI (New England Biolabs, Ipswich, MA, USA) digestion at $37^{\circ} \mathrm{C}$ overnight. $5^{\prime}$ VNTR was amplified in reported primers [38] through 40 cycles of $94^{\circ} \mathrm{C}, 30 \mathrm{sec}, 60^{\circ} \mathrm{C} 30 \mathrm{sec}$ and $72^{\circ} \mathrm{C} 45 \mathrm{sec}$. All DNA fragments were resolved by electrophoresis in $1.1 \%$ agarose gels.

3. Haplotyping of DNPi and 5'VNTR was performed by using SHEsis [39].

4. Statistical analyses were done using SPSS Statistics Subscription version 26 (IBM, Armonk, New York, USA). Multivariate analyses were performed with genotype/haplotype as the between-group variable and hDAT availability in striatal subregions as dependent variables.
For $5^{\prime}$ VNTR, repeats were combined as a quantitative variable. Age and gender were added as covariates. Post hoc univariate tests and $t$ tests were performed to investigate directions of effects. $P<0.05$ were considered statistically significant.

Postmortem mRNA density of hDAT in laser-capture microdissection (LCM)-isolated postmortem DA neurons from 20 healthy subjects had been previously estimated [40] and its correlation with DNPi genotype is presented in Insert of Fig. 3 upper panels.

\section{Phylogenomics \& intragenic epistasis analyses}

Polymorphism information in the 26 1KGP populations was used to generate phylogenic trees via ClustalX and TreeView [41, 42]. Intragenic epistases (case-control epistatic associations) were evaluated by metaanalyses of logistic regression results, as previously described [43] except only focusing on Caucasians in this study (the African American cohort was excluded). $P_{\text {meta }}<0.05$ from Bonferroni-based multiple-testing was considered as statistically significant. Data are described in Fig. 4 and in Supplementary Table 1.

IDRs of TFs prediction was carried out using the MobiDB software [44] and the data are presented in Supplementary Table 2 in the Supplementary Information.

\section{RESULTS AND DISCUSSION}

\section{Genetic associations}

To reveal genetic risks in SLC6A3, two types of association studies have been carried out, familial mutations and case-control associations.

Familial mutations. During the last decade, 26 hDAT mutants have been identified mainly in individuals with dopamine transporter deficiency syndrome (DTDS) [45] (Table 1) [46-64].

The first causal relation between functional deficiency of hDAT and a clinically relevant disorder was reported by us in 2009 [47] with family members carrying an homozygous mutation (Leu368GIn or Pro395Leu) affected with dopamine transporter deficiency syndrome (DTDS) whereas family members that were wild-type or heterozygous carriers did not show the clinical syndrome of DTDS. This appropriate segregation of mutation with disease status was observed for all of the subsequent DTDS cases studied by us, indicating causality [45] (Table 1 cases 1-11, 16-19) [46-64]. Typical DTDS patients display dramatic infantile phenotypes with progressively worsening dystonia and parkinsonism and are at risk of premature death in childhood or adolescence. Upon arranging all cases according to the severity of functional DA uptake, it can be seen that the first 19 mutants in the Table lost $100-70 \%$ reuptake activity, characteristic of DTDS except case\#15 ( $\triangle$ N336) with autism (see Fig. 2a legend). Some patients display atypical later onset parkinsonism (case\# 18 and see Fig. 2a), with one patient additionally afflicted with ADHD (case\# 20) [54].

Six mutations were implicated in mental illnesses only: Arg51Trp [63], Thr356Met [57], Ala559Val [60] and $\Delta$ N336 [52] in autism, Arg615Cys in ADHD [58], and Glu602Gly in bipolar disorder (BP) [62]. Thr356Met drove persistent reverse transport of DA (efflux) [57]; $\triangle N 336$ displayed impaired DA transport, reduced $A M P H$-induced DA efflux, diminished AMPH-induced currents, resulting in autism-related behaviors in Drosophila. Arg615Cys might increase membrane mobility [65]. Although there is no evidence that these mutations cause the observed mental illnesses, the rarity of the former mutations and their coincidence with a psychiatric disorder does suggest their role as a risk factor.

These mutations are located throughout the protein structure (Fig. 2a left panel), with the DTDS mutations mostly in the translocation path for reuptake activity, ion binding and/or surface localization (Fig. 2a right panel), explaining their impaired reuptake. The mutations associated with psychiatric conditions 
a
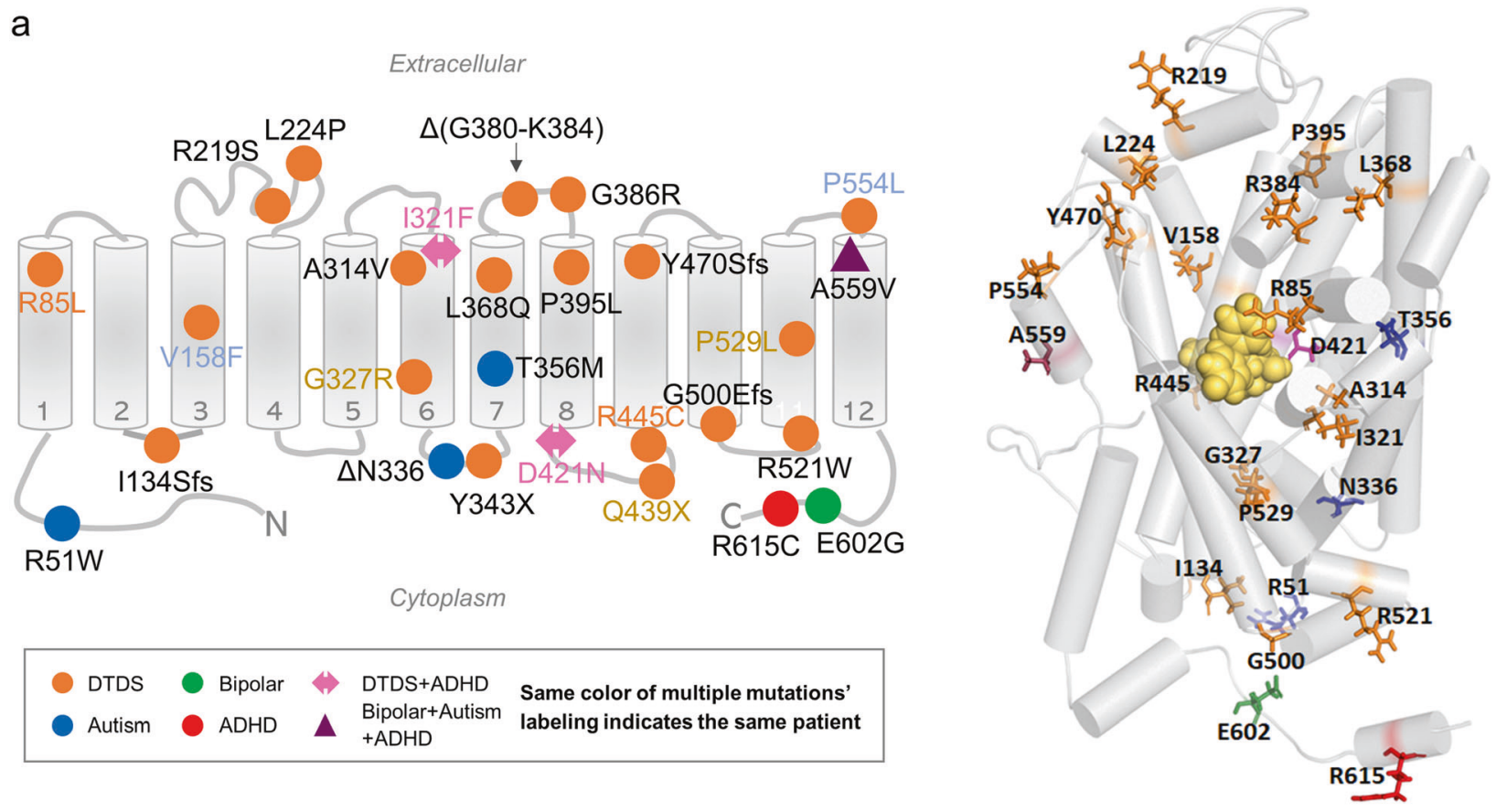

b

9r/10r

epression

PTSD

Smoking

cessation

Cytoplasm

\begin{tabular}{|lllll}
\hline DTDS & Bipolar & $\Leftrightarrow$ & $\begin{array}{l}\text { DTDS+ADHD } \\
\text { Bipolar+Autism } \\
\text { Autism }\end{array}$ \\
+ADHD
\end{tabular}$\quad \begin{aligned} & \text { Same color of multiple mutations' } \\
& \text { labeling indicates the same patient }\end{aligned}$

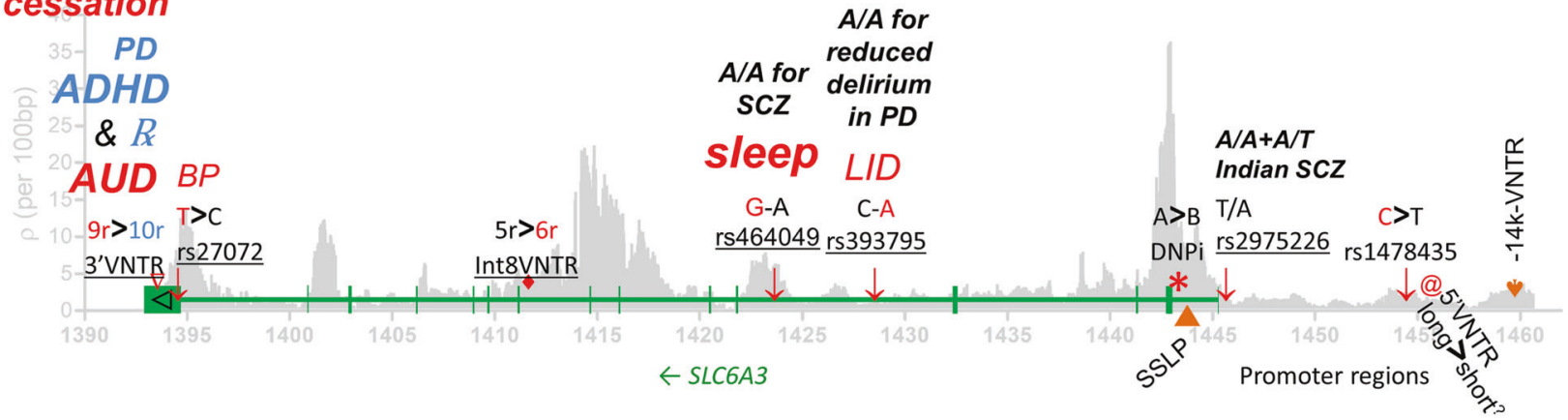

Fig. 2 Functional syntheses for association signals. a Molecular modeling of familial hDAT mutants for structural interpretation. (left) Twodimensional distribution of mutations. Each gray cylinder represents a transmembrane (TM) domain; upside, synaptic space; downside, cytosol of DA neuron. (right) Three-dimensional structural model of mutated residues with TM helices represented by gray cylinders. Mutated residues are represented as licorice sticks and colored using the same convention as in (left panel): DTDS in orange, autism in blue, ADHD in red, BP in green and the combined mutants for autism, BP and ADHD in magenta and labeled. The substrate/inhibitor site that includes both S1 and S2 sites is represented by the docked inhibitor represented in space filling model and colored yellow (these represent new information - see Supplementary text for Fig. 2a in the Supplementary Information). b Associated (underlined), functional (>) or other complex (orange symbol) markers in regulated SLC6A3. SLC6A3 has 15 exons (14 introns), with the start codon in Exon 2 and the stop codon in Exon 15 so that its $1863 \mathrm{bp}$-coding region ends with the first $23 \mathrm{bp}$ of the $2 \mathrm{~kb}$ long Exon 15 (based on GenBank Accession \# NM_001044). Indicated are functional markers in red and other unique complex markers in orange: 3'VNTR and rs27072 in 3'UTR, Int8VNTR, rs64049 and rs393795 in Intron 4, DNPi and SSLP ( $\mathbf{A})$ in Intron 1, rs2975226 in core promoter, rs1478435, 5'VNTR (@) and -14k-VNTR (v) in distal promoter regions. SNPs are indicated with $\downarrow$. Green, SLC6A3 gene structure (vertical bars are 15 exons in the opposite strand of the chromosome); $>$, variant activity greater than; -, unknown activity for two alleles; /, a genotype associated; italics, associated diseases or response to medication (R) (BP bipolar, METH methamphetamine use disorder, LID levodopa-induced dyskinesia, SCZ schizophrenia); bold, meta-analysis result and font size indicates significance or sample size used where same color matches risk allele with phenotype;?, only postmortem mRNA correlational data; gray: recombination rate (new data) obtained from combining all 25 1KGP populations, below with the chr5 position in kb. Paucity, lack of association efforts in promoter regions (no familial mutants are included). Only association studies with meta-analysis statistical significance or reproduceable results are summarized here.

affect DA efflux and involve interactions with other proteins such as $G \beta Y$ subunits of the G-protein for Glu602Gly in BP and Arg615Cys in ADHD or syntaxin for Arg51Trp in autism [66, 67], affecting hDAT networking (see Supplementary Text for Fig. 2a). However, it remains unknown whether some of the phenotypes are also attributable, in part, to associated synonymous variations.
Case-control associations. Classic markers used were two variable number of tandem repeats (VNTRs), one in the $3^{\prime}$ untranslated region (3'UTR) commonly known as 3'VNTR of 40 bp (rs28363170), and another in Intron 8 or Int8VNTR of $30 \mathrm{bp}$ (rs3836790). 3'VNTR has two common alleles, 9- and 10-repeat $(9 r, 10 r)$ and Int8VNTR also has two common alleles, 5- and 


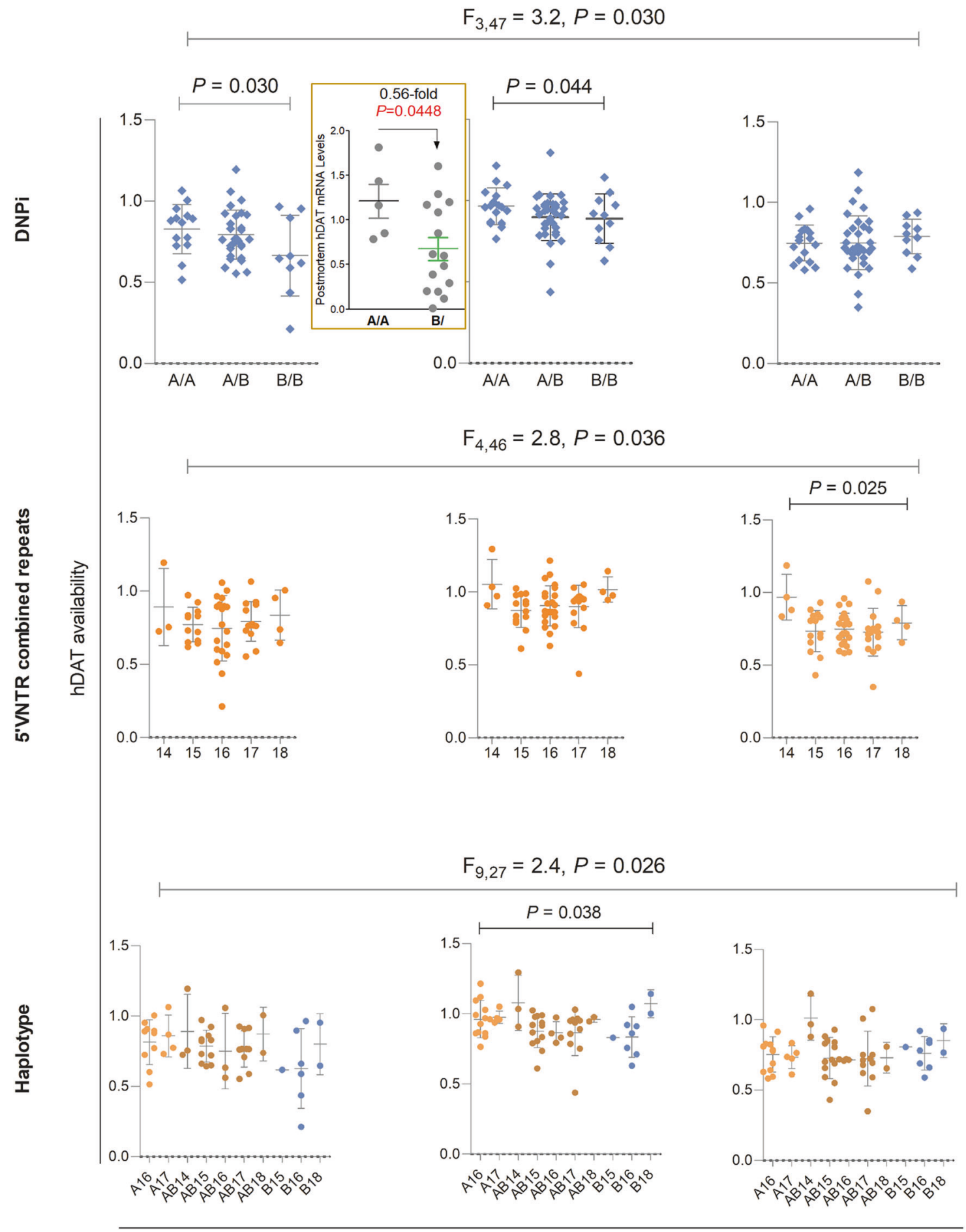

genotype/haplotype

Fig. 3 Genotype-correlation of hDAT availability (Bmax/KD in $Y$ axis) in healthy ventral striatum (left), putamen (center) or caudate (right). Insert, postmortem hDAT mRNA levels in isolated nigral DA neurons from twenty independent healthy subjects; upper, DNPi; middle, $5^{\prime}$ VNTR; bottom, haplotype of DNPi and 5'VNTR; multivariate results for three brain regions are given in $F$ values and only significant $P$ values are shown $(N=62)$. Between-genotype differences were not statistically significant after Bonferroni corrections. After excluding women ( $N=$ $3)$, the effects remained for men. These are all new data. 
a. $18 \mathrm{~kb}$ Promoter

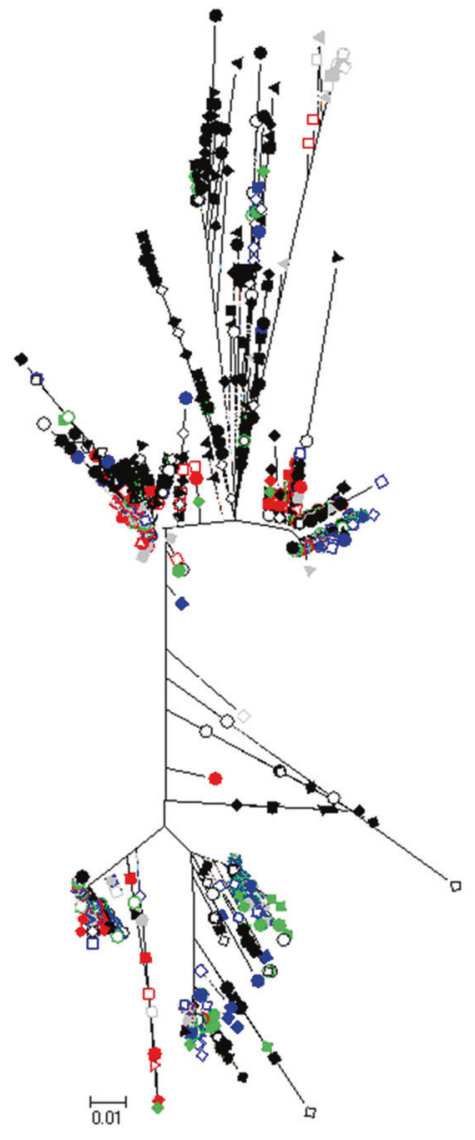

b. $70 \mathrm{~kb}$ Entire gene

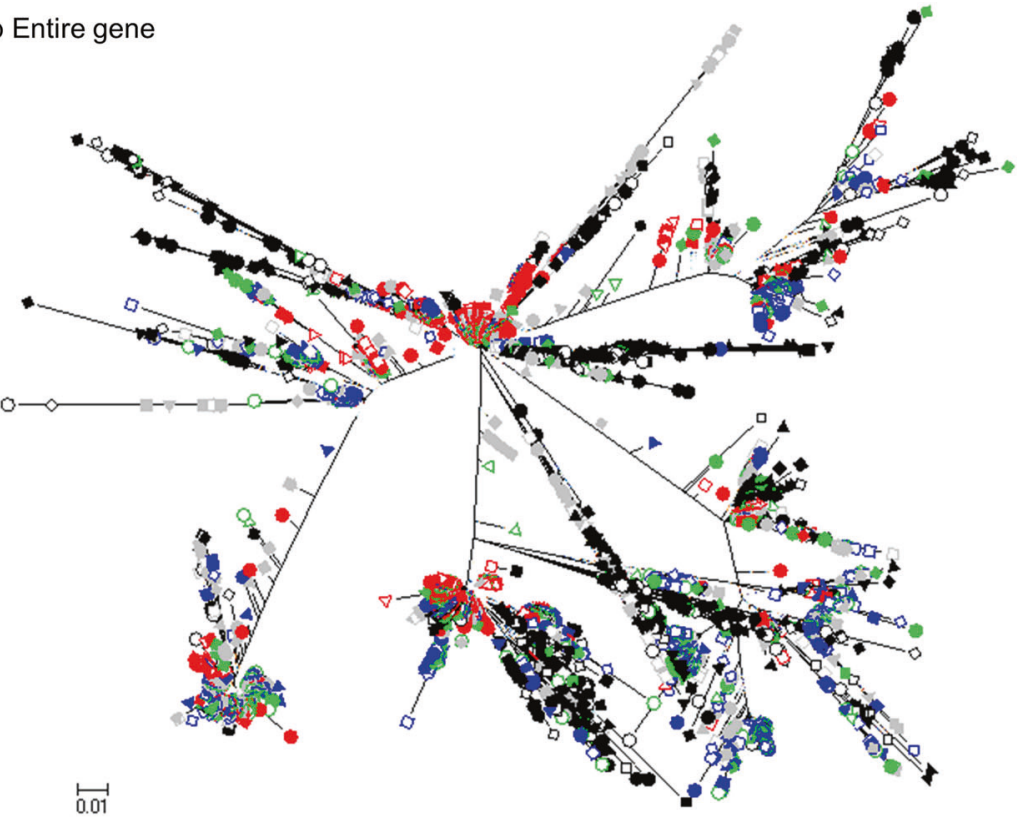

c. Intragenic epistasis within $70 \mathrm{~kb}$ (including $18 \mathrm{~kb}$ promoter)

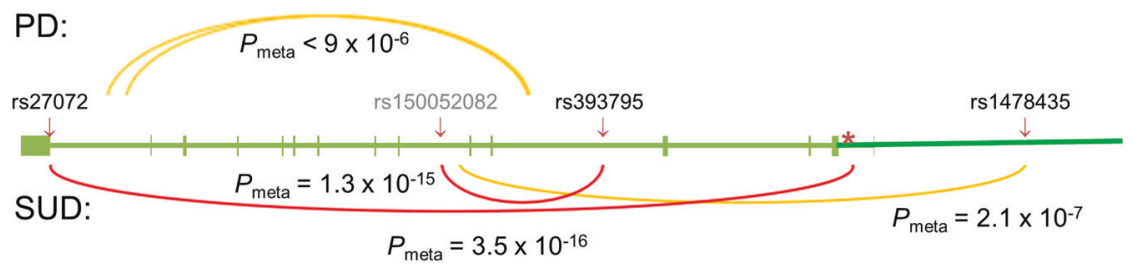

Fig. 4 Genetic evidence for functional variants throughout $S L C 6 A 3$, based on haplotype selection of $18 \mathrm{~kb}$ promoter (a) or $70 \mathrm{~kb}$ whole gene (b) and on case-control intragenic epistases for PD and SUDs both in Caucasians (c). In first two panels of phylogenic trees, labeling of the 26 1KGP populations are explained as follows. East Asians all in red: closed circle for Chinese Han Beijing (CHB); open square, Japanese (JPT); closed square, Chinese Han South (CHS); closed diamond, Chinese Dai in Xishuangbanna (CDX); open triangle, Kinh in Chi Minh City, Vietnam (KHV). European ancestry all in blue: closed circle, Utah residents with Northern and Western ancestry (CEU), open diamond, Toscani in Italia (TSI); closed triangle, Finnish in Finland (FIN), closed square, British in England and Scotland (GBR); open square, Iberian population in Spain (IBS). African ancestry all in black: closed circle, Yoruba in Ibadan, Nigeria (YRI); closed diamond, Luhya in Webuye, Kenya (LWK); closed triangle, Gambian in Western divisions of the Gambia (GWD); inverse closed triangle, Mende in Sierra Leone (MSL); closed square, Esan in Nigeria (ESN); open circle, Americans of African Ancestry in SW USA (ASW); open diamond, African Caribbeans in Barbados (ACB). Admixed Americans all in green: closed circle, Mexican ancestry from Los Angles USA (MXL); closed diamond, Puerto Ricans in Puerto Rico (PUR); open circle, Colombians in Medellin of Colombia (CLM); open triangle, Peruvians in Lima of Peru (PEL). South Asian all in gray: closed circle, Gujarati Indian in Huston of Texas USA (GIH); closed diamond, Punjabi in Lahore of Pakistan (PJL); closed square, Bengali of Bangladesh (BEB); open square, Sri Lankan Tamil from the United Kingdom (STU); and closed triangle, Indian Telugu from the United Kingdom (ITU). In panel (c), 6572 unrelated subjects in three cohorts were used for PD (above the gene schematic related to Fig. $2 \mathrm{~b}$ ) and 5843 in another three cohorts, for SUDs (below the gene schematic). Shown are intragenic epistases selected largely for those involving Fig. 2b-mentioned markers where yellow indicates suggestive significance and red, for statistical significance. Unlabeled SNPs are rs11564770 (at 1398806) and rs11564772 (at 1398007) in Intron 14 or last intron, and rs250686 (at 1425159) in Intron 4 for PD; rs11564757 (160 bp downstream of DNPi indicated by asterisk) in Intron 1 and rs2975292 (at 1419932) in Intron 6 for SUDs. rs150052082, in gray, was a new SNP which interacted with multiple loci in SUDs, see Supplementary Table 1 for details. These are all new data.

6-repeat $(5 r, 6 r)$. Different populations may have additional alleles in either case. Moreover, several single nucleotide polymorphisms (SNPs) in various regions have also been used as association markers. Considered here are only reproduced and statistically significant findings, as summarized in Fig. 2 b. SNP-based genome wide association studies (GWAS) have not implicated SLC6A3 in the neuropsychiatric diseases [68-70]. This was likely due to ethnicity or the fact that GWAS were based on SNPs and the associations with ADHD and alcohol use disorder (AUD), for example, are on 5'VNTR, not SNPs, given the high recombination rates between $5^{\prime}$ VNTR and SNPs in other regions of this gene (see Fig. 2b).
A. Etiology. Most studies focused on diseases related to DA transmission in the CNS, based on a priori knowledge.

Substance use disorders (SUDs) AUD and tobacco use disorder (TUD) are two leading causes of chronic diseases so they became a main focus of SLC6A3 association studies. In 2016, the Li Lab published two meta-analyses. One was on AUD in 5846 participants, concluding that $3^{\prime}$ VNTR9r, the shorter allele, was the risk variant [71], which has been further supported by more recent findings $[72,73]$. The other was on smoking cessation in 5480 participants, concluding that $9 \mathrm{r}$ was a promoting variant for cessation (pooled $O R=1.17$ ) [74], which has been further supported by recent studies in 819 Chinese and 1230 Russian 
I

导

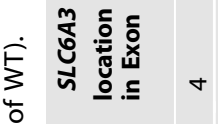

o

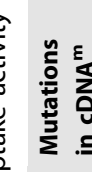

$\frac{\hat{\hat{o}}}{\mathrm{o}}$

$\overleftarrow{\Delta}$

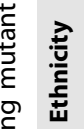

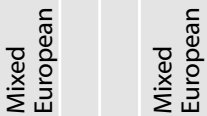

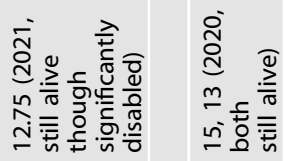

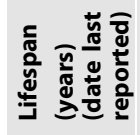

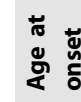

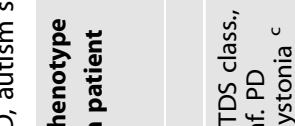

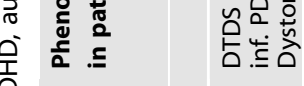

定

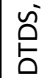

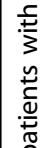

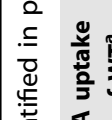

表

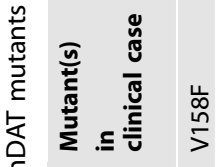

高

产

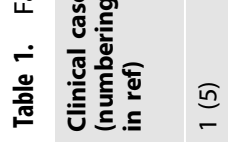

$\frac{\sqrt{0}}{\frac{2}{0.0}}$

$\begin{array}{llll} & & \stackrel{n}{n} \\ m & \infty & 0 \\ 0 & 0 & 0 \\ 0 & 0 & 0 & 0\end{array}$

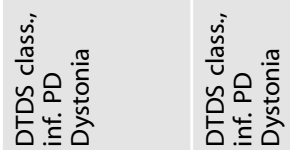
능

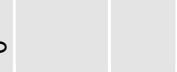

$\circ \circ$

志

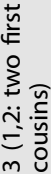

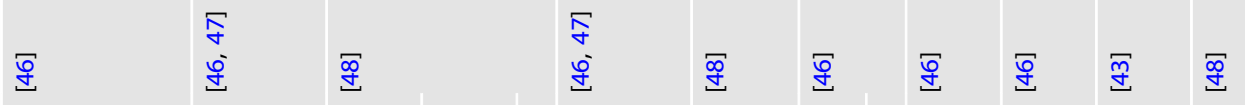

들ำ

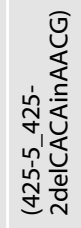

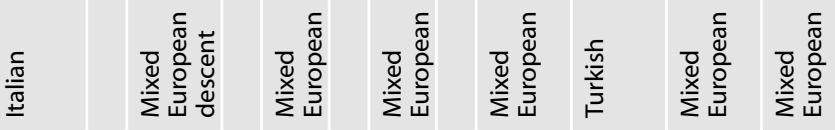

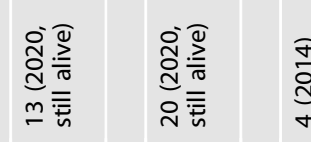

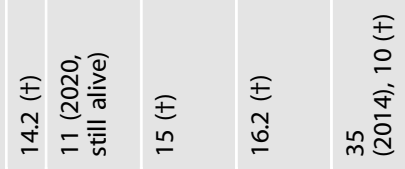

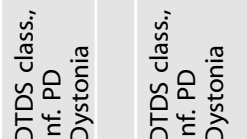

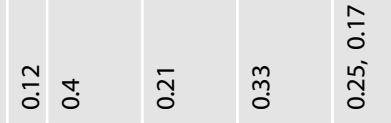

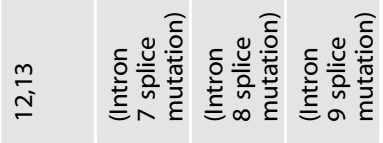

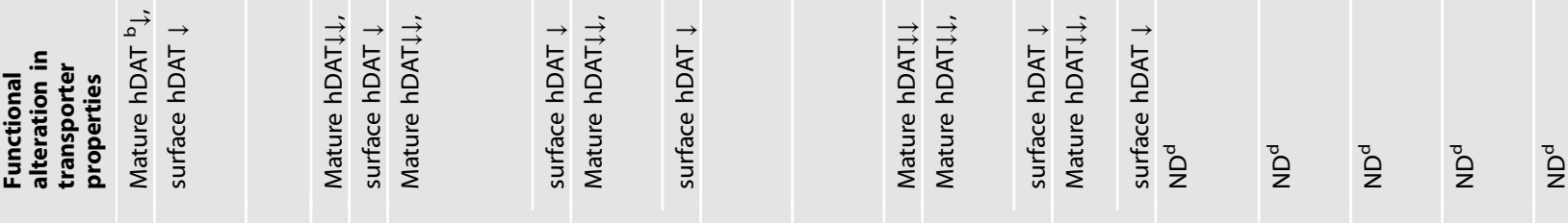

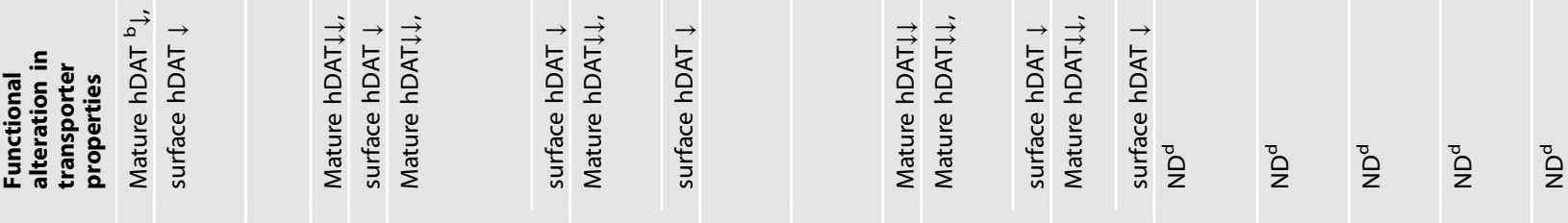

$\circ \quad \stackrel{\circ}{0}$

$00 \quad 0 \quad \frac{0}{\bar{a}} \quad \frac{0}{\bar{a}} \quad \frac{0}{\bar{a}}$

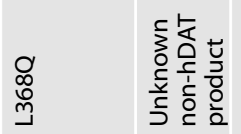

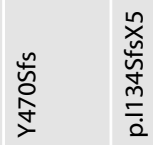

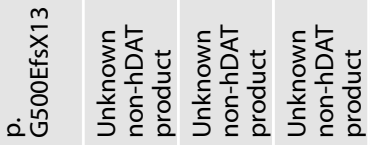

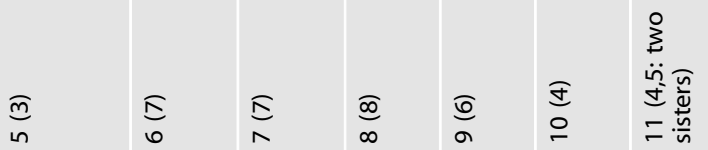


I হ্

둥

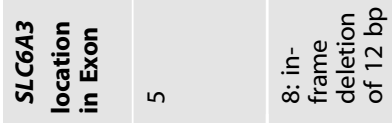

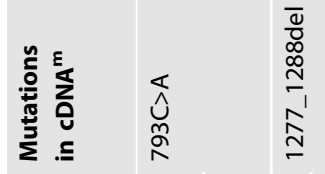

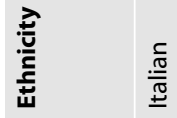

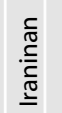

迹离

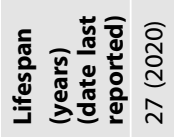

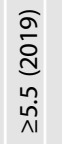

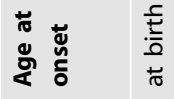

$\frac{1}{0}$

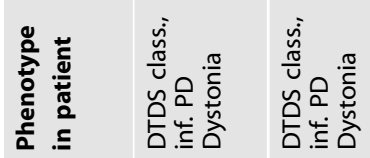

ह స్․․

$\wedge \quad \wedge$

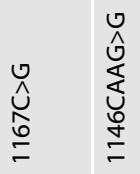

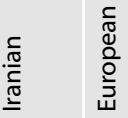

$\Sigma \quad \Sigma$

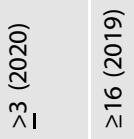

$\stackrel{m}{0} \quad \frac{\circ}{v_{1}}$

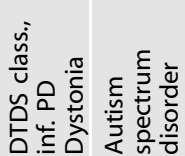

导

$\wedge$ 우

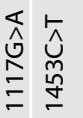

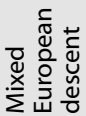

\llcorner

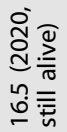

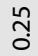

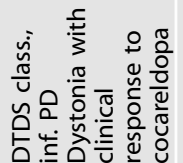

लำ

㐫

으

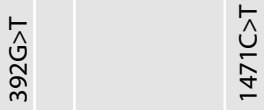

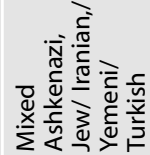

实

$\stackrel{n}{\stackrel{n}{o}}$

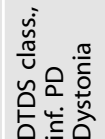
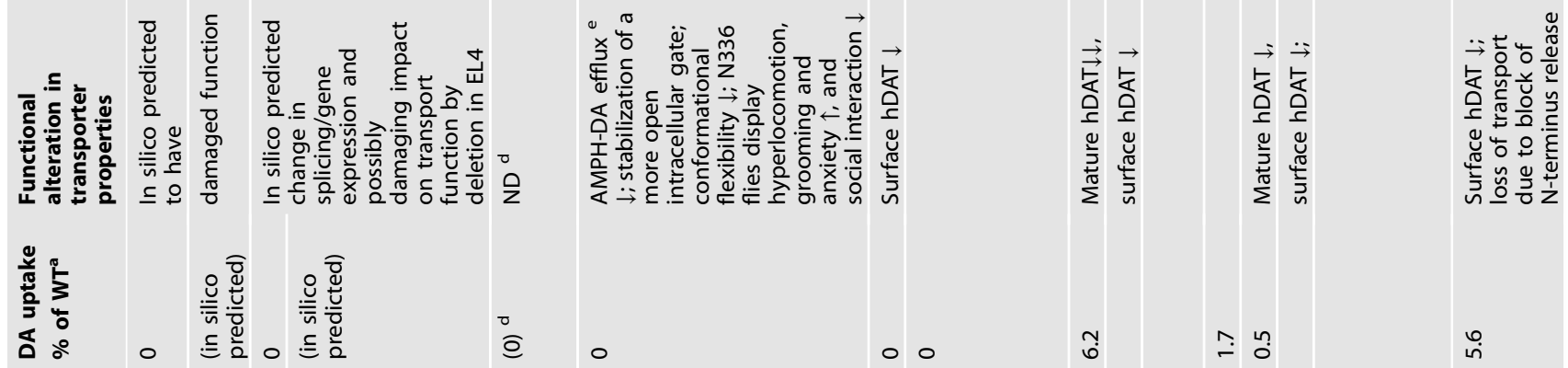

00 กูก

i̊

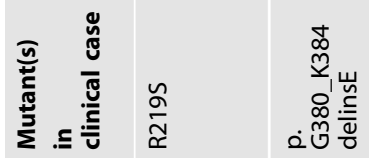

\begin{tabular}{l|l}
$\underset{m}{n}$ & $\stackrel{m}{m}$ \\
$\stackrel{m}{j}$ & $\triangleleft$
\end{tabular}

舜舜

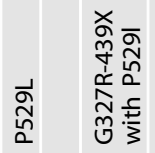

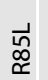

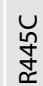

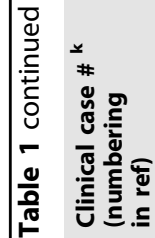


I

III

II

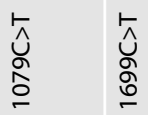

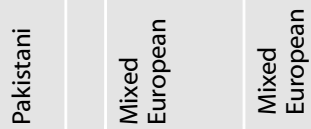

竞

ปัँ

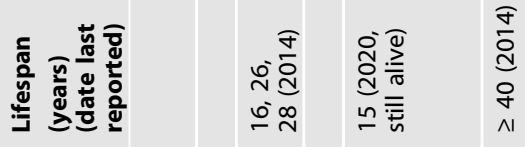

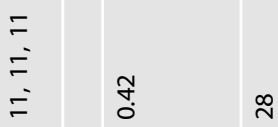

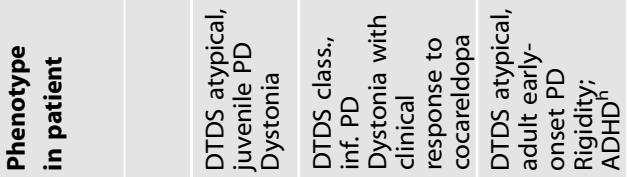

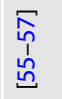

$\infty$

苍

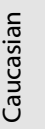

$\Sigma$

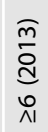

vi

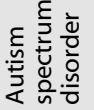

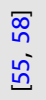

ฮ్

$\stackrel{n}{\llcorner}$

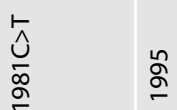

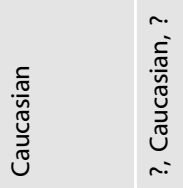

$\Sigma \quad \sum_{n=}^{n}$

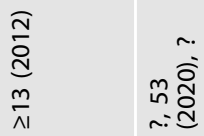

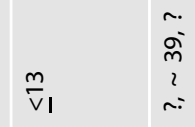

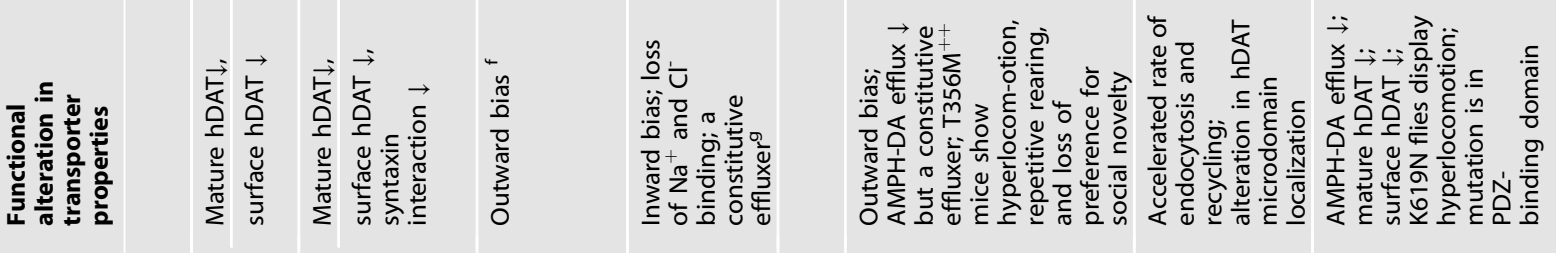
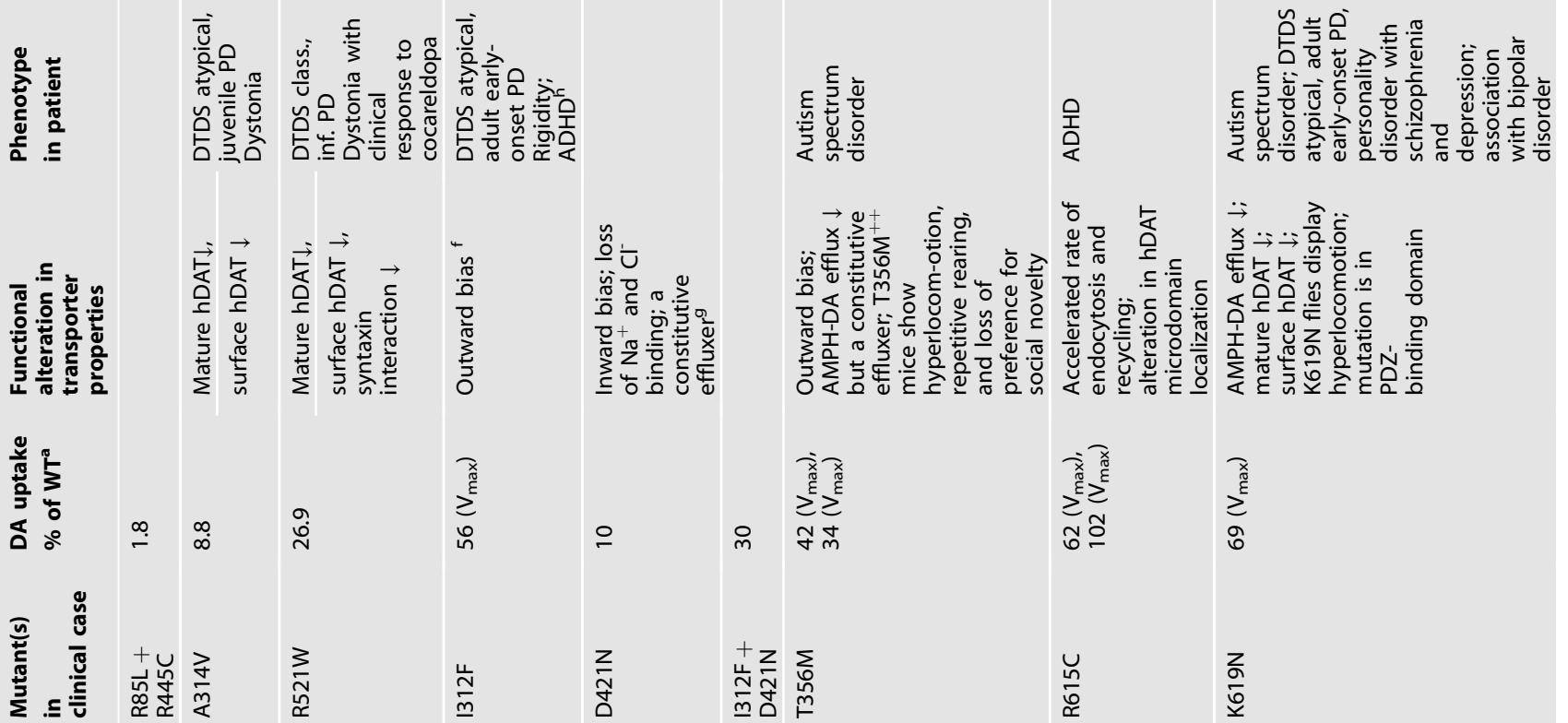


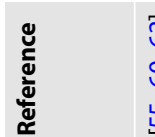

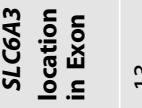

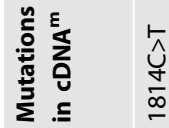

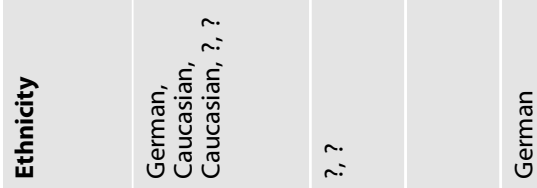

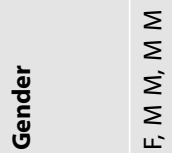

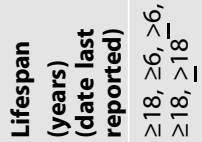

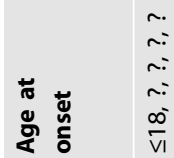

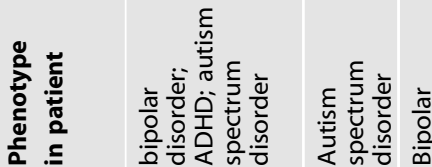

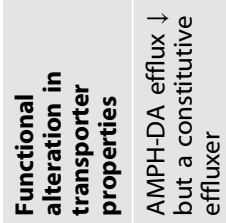

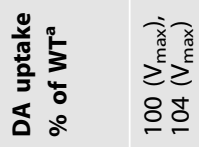

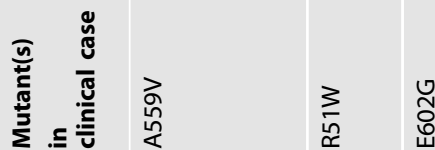

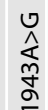

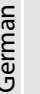

$\stackrel{\infty}{\mathrm{V} I}$

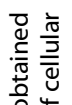

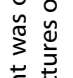

究

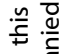

흔

ลे प्र

낭

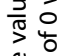

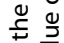

永垔

要焉

ํํㅇ

它

嵌

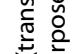

品

跣

仓ิ่

둥

흔

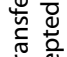

贾

垔

空

妾

琹

气્山

⿵人一

焉家

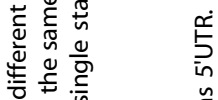

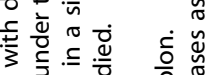

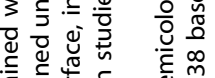

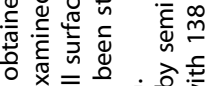

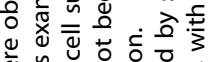

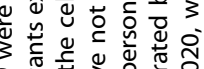

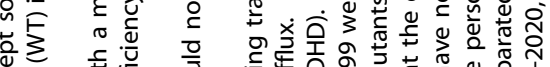

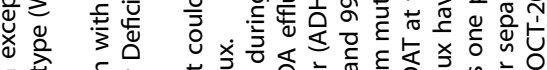

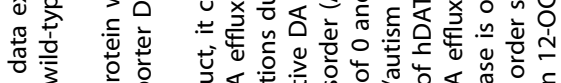

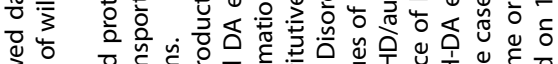

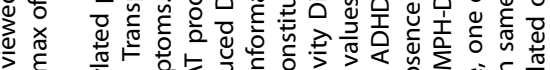

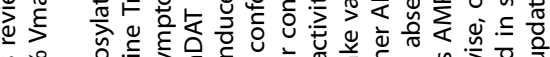

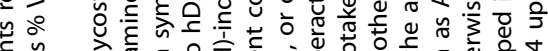

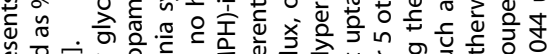

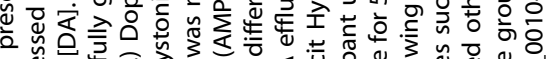

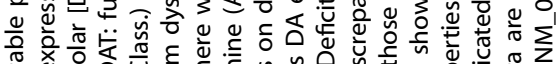

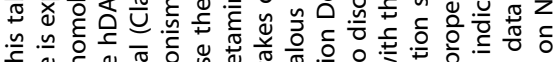

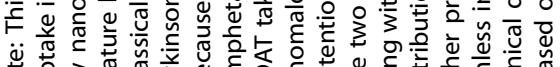
Min 
1040

subjects $[75,76]$. Therefore, both in AUD and TUD the $9 r$ variant is a risk allele; a remaining question is whether this variant has a role in relapse vulnerability.

ADHD: A meta-analysis of 59 studies on $3^{\prime}$ VNTR among a total of 31,457 children and adolescents with ADHD indicated that $10 \mathrm{r}$ is the risk allele [77]. The association was significant in Caucasians and in Europeans, but not in Asians.

Other complex psychiatric diseases: Studies employing metaanalyses have implicated this gene in posttraumatic stress disorder (PTSD) (3'VNTR9r OR = 1.62) [78], MDD (pooled OR= 1.26) $[79,80]$, schizophrenia $(S C Z)$ (pooled $O R=3.2$ ) [81], sleep duration (rs464049 G allele, beta [standard error, SE] $=-0.96$ [0.18] minutes/allele; $P=5.71 \times 10^{-10}$ as a genome-wide significance) [82] and PD [83]. An early Dutch study examined 16 SNPs plus 3'VNTR in 720 patients and uncovered selective rs393795 A/A protection against delirium in PD [84]. The study's meta-analysis of three SNPs in 1641 patients indicated consistently and selectively that rs393795 A/A reduces delirium in PD $(O R=0.37)$ [85]. This gene apparently contributes to PD in multiple ways. In addition, rs27072, a SNP in 3'UTR, was implicated in BP [86]. Even though psychiatric diseases are multifactorial, all these studies point to an important risk coming from the 9- and 10-repeat alleles of the 3'VNTR of SLC6A3.

$B$. Response to medications. Several studies reported genetic effects of SLC6A3 on response to medications. A meta-analysis of 36 studies with a total of 3647 youth for genetic modulations of methylphenidate's efficacy in ADHD treatment found that in 16 studies, the ADHD-risk variant 3'VNTR10r was associated with reduced efficacy $(O R=0.74)$ [87]. Clearly, taking into account such individual genetic variability may improve the success of childhood-ADHD treatment with methylphenidate (Ritalin).

Another pharmacogenetic involvement was found for levodopa in PD patients. Two recent reviews agreed that SLC6A3 contributed to the response to levodopa in various cohorts $[88,89]$. For example, results in 352 levodopa-treated Israeli PD patients indicated that the C allele of rs393795 extended significantly the time to levodopa-treated dyskinesia (LID) onset, time ratio $=4.96\left(95 \% \mathrm{Cl} 2.3-10.9, P=4.1 \times 10^{-5}\right)$ [90]. Findings from recent studies further supported the association of this SLC6A3 variant with LID [90, 91]. Moreover, SLC6A3 may interact with other DA concentration-regulating genes (SLC18A2 and COMT) in treatment-related complications [92]. Although the risk of LID is a consequence of a number of intrinsic (patient-related) and extrinsic (medication-related) factors, these studies point to an important contribution to the risk by genetic effects of SLC6A3.

\section{Common regulatory variants}

To clarify disease mechanisms, the first step is to investigate which variants display altered activity or respond to a regulator. More than 10 polymorphisms have been identified as risk variants in association studies but only three of them have been assessed for in vitro cis-acting or transcriptional regulatory function.

\section{Three known polymorphisms}

3'VNTR cis-activity: 9r is higher than 10r: 3'VNTR, the first SLC6A3 marker used, has been the most studied, followed by Int8VNTR. Two groups reported consistently that 3'VNTR9r enabled higher in vitro CMV promoter activity than 10r [93, 94], which is supported by studies in HEK293 cells that showed $9 \mathrm{r}$ to display higher promoter activity than the $10 \mathrm{r}$ allele; however, both showed lower activity than the control (without the VNTR), suggesting the 40bp-repeat is cis-inhibitory [95].

Imaging studies in humans can clarify the relationship between $3^{\prime}$ VNTR and hDAT abundance. Many disease conditions, including SUDs, can affect gene activity and disrupt this relationship so studies must first evaluate the relationship in healthy individuals. A meta-analysis of imaging findings from 12 studies in a total of 511 individuals reported that the $9 \mathrm{r}$ variant correlated with increased hDAT protein level in healthy individuals by either PET or SPECT but this relationship was attenuated in affected individuals (patients with ADHD, AUD, PD and SCZ) [96]. The latter suggests that there is an additional, unknown factor that comes into play after the disease has developed, i.e. the risk from $S L C 6 A 3$ is important in developing the disease.

Int8VNTR cis-activity: $5 r$ is higher than $6 r$ : Studies of in vitro heterogeneous promoter activity in SH-SY5, reported that the shorter allele $5 r$ displayed higher hDAT activity activity than the longer allele $6 r$ [97]. This data was consistent with a finding from a reporter analysis in the mouse substantia nigra-derived dopaminergic cell line SN4741 [98].

We have clarified in humans a relationship between Int8VNTR and hDAT abundance by PET imaging of 95 healthy subjects with the consistent result that $5 \mathrm{r}$ was associated with higher hDAT availability than $6 r$ [99].

The above studies utilized heterogenous promoters, not the human promoter, for the allelic functions. We have looked at the allelic effects by combining the shorter alleles or combining the longer alleles on the entire human SLC6A3 promoter in $18 \mathrm{~kb}$ of two haplotypes ( $A$ and $B$ ); we found that the human promoter carrying the $9 r+5 r$ together displayed higher promoter activity than one carrying the $10 r+6 r$ together, especially in the $18 \mathrm{~kb} \mathrm{~B}$ haplotype and in SN4741 [100].

PET studies of healthy individuals confirmed independently that $10 r+6 r$-carriers had the lowest hDAT availability [99], establishing that the shorter alleles confer higher promoter activity than the longer alleles under basal conditions. Given the findings that these VNTRs both are inhibitory [100], it is plausible that the shorter alleles confer higher promoter activity by carrying less inhibitory activity with the smaller number of repeats.

rs27072 cis-activity in $3^{\prime}$ UTR: T is higher than C: Rs27072 is a common variant with an average minor allele frequency (MAF) of around $20 \%$ by 1 KGP. In a BP association study Pinsonneault et al. [86] assessed postmortem mRNA levels, used an in vitro promoter reporting assay, and measured DA uptake activity; the results indicated that the minor allele $\mathrm{T}$ is a risk variant, displaying an enhanced hDAT activity.

Three new markers around the promoter. We recently reported several novel and common polymorphisms around the promoter regions $[38,101]$ and three of them already have functional implications.

DNPiA-B (rs67175440) cis-activity in humans: A is higher than $\mathrm{B}: \quad \mathrm{DNPi}$ (dinucleotide polymorphism in Intron 1) has two alleles $A$ and $B(A \bar{A} \bar{A} G, B: G A$ as two adjacent bases of rs2975223T/C and rs2937640C/T) [38], a very common variant with an average MAF of 39\% (12-50\%, 1KGP). A long non-coding RNA (IncRNA, termed AZI23'UTR) DNPiB (rs2975223T) allele-dependently inhibited the SLC6A3 promoter activity in vitro related to SUDs [102], suggesting DNPiB is a new functionally inhibitory variant.

PET imaging re-analysis showed an overall multivariate effect of DNPiB rs2975223 on hDAT availability $\left(\mathrm{F}_{3,47}=3.2, P=0.030\right)$. Posthoc comparisons indicated elevated hDAT availability in the DNPiB rs $2975223 \mathrm{~A} / \mathrm{A}$ group compared to $\mathrm{B} / \mathrm{B}$ in putamen $(P=0.044)$ and ventral striatum $(P=0.030)$. hDAT availability was also higher in ventral striatum for $A / B$ than $B / B$ at trend level $(P=0.056)$. These protein-based imaging findings were confirmed by mRNA results in single postmortem nigral DA neurons (Fig. 3 upper panels).

5'VNTR (rs70957367) cis-activity: possibly more repeat, more activity: $5^{\prime}$ VNTR is located $11 \mathrm{~kb}$ upstream of Exon 1 and has 
60 bp for 6-9 repeats [38]. Postmortem mRNA data has suggested it to be a functionally related marker, with a combined repeat number of two alleles from 15 to 18 positively correlated with the mRNA levels in controls, but not in cocaine abusers [38]. This finding suggests that $5^{\prime}$ VNTR may function as an enhancer.

PET imaging re-analysis showed an overall multivariate effect of $5^{\prime}$ VNTR on hDAT availability $\left(F_{4,46}=2.8, P=0.036\right)$. Univariate analyses showed this effect was present for caudate $\left(F_{4,52}=3.1, P\right.$ $=0.025$ ), mainly due to higher levels in 14-repeats-carriers (Fig. 3 middle panels). The 15-18 repeats showed a consistent tendency of more repeats, more activity and in fact, post hoc $t$ tests showed a significant group difference between 15 and 18 in putamen $(P=$ 0.042 ). The main group differences were largely due to the combined 14-carriers.

Haplotype analysis of DNP1 and $5^{\prime}$ VNTR still suggested a genetic effect on hDAT availability $\left(F_{9,27}=2.4, P=0.026\right)$, especially in putamen $(P=0.038)$ (Fig. 3 bottom panels). These statistical significances disappeared after Bonferroni correction.

rs1478435 C-T cis-activity: $C$ is higher than T: Located at $9057 \mathrm{bp}$ upstream of Exon 1, rs 1478435 is a common variant with MAF of $26 \%(\mathrm{~T})$ per 1KGP. The $C$ allele carries an enhancing activity on the SLC6A3 promoter, related to SCZ [40]. By contrast, the T allele was associated with reduced gene activity based on reduced mRNA levels in postmortem nigral DA neurons from healthy individuals and reduced transcriptional activity based on $2.5 \mathrm{~kb} S L C 6 A 3$ promoter reporting assays in two cellular models. We have not looked at it in human subjects yet.

These new and verified $5^{\prime}$ markers may allow us a superior ability to detect association signals. In addition, a simple sequence length polymorphism (SSLP) in Intron 1 and a -14kb-VNTR represent two novel common markers [101], worthy of functional investigation. Collectively, state of the art risk information including allelic activity under basal conditions is now aligned with the association signals in Fig. $2 \mathrm{~b}$.

\section{High vs low activity variant as a risk}

Several studies [103, 104], including ours [40, 105, 106], have shown increased SLC6A3 activity associated with brain disorders such as AUD, PTSD, BP and SCZ except ADHD or smoking cessation. In fact, chronic DA depletion is noticed already as an underlying factor for SUDs [107-110], which can be a result of increased SLC6A3 expression or a reduced dopaminergic tone. Consistently, known environmental risks such as the most established risks stressors and nicotine could increase Slc6a3 activity in vivo [19, 111-113].

Like tobacco smoking, cannabis use in adolescents may become a gateway by increasing the vulnerability for SUDs including AUD and OUD in later life [114-116]. Such gateway effects may occur through adaptation processes in DA neurons [117]. Interestingly, cannabinoid receptor type 2 (CB2) is expressed in DA neurons and modulates cannabis' addictive effects [118]. Deletion of CB2 from DA neurons reduced alcohol preference [106] and Slc6a3 activity [119] in mice, suggesting that cannabis' effects may be mediated through the activation of SLC6A3 but also that activation of SLC6A3 may be part of the adaptation processes. This information again supports the view that increased SLC6A3 activity could be a risk for SUDs.

A recent study reported a decreased hDAT level associated with depression [120]. However DAT knockdown reduced anxiety and depression-related behaviors in mice [121, 122], supporting a view that reduced hDAT levels protect against depressive behaviors. Such contradicting results suggest the possibility that the clinically observed decrease could be a result rather than a cause of the disease, representing another example for disease effects on gene activity.

Decreased gene activity can be a risk too, for diseases like Caucasian ADHD. These findings are not surprising considering ethnic medicine and polygenetic disorders. It is noted that a simplistic mode of action such as up- or down-regulation is unable to explain various association findings, warranting investigation of the dynamics of hDAT regulatory networks.

\section{Choice of markers}

Given the fact that there are $>2500$ SLC6A3 SNPs per 1KGP, design of a case-control association study needs to evaluate markers for three reasons.

First, many markers have no functional implication yet, including whether any risk pathways can regulate them in an allele-specific manner. If they are not the underlying variants, the association signals could be linkage disequilibrium (LD)-guided results. SLC6A3 has many recombination hotspots, which occur among the common markers and separate the downstream VNTRs from the promoter regions (Fig. 2b) [101]. For a solid association signal, it is important to use the underlying functional marker or a marker close to or of strong LD with the underlying locus.

Second, given multiple functional variants, it comes down to whether all need to be typed especially when some are enhancing activities and others are inhibitory. This is because there could be intragenic antagonisms among these loci [123-125] so that use of one or few of them could be a biased study design. Regulation has only two end directions, up and down, regardless of the number of functional loci present but the key is its dynamics, when and what regulate the gene via these loci, partly defining the multidisease mechanism. Therefore, multimarker association analyses may enable gene-wide delineation of disease and comorbidity mechanisms.

The third reason is a technical aspect since some complicated markers are difficult to work with and impossible to type with a high throughput manner. SLC6A3 has such complicated markers, which include the SSLP at +1531 in Intron 1 (nine known alleles) and the variable but complicated sequence arrangement $-14 \mathrm{~kb}$ VNTR (four known common alleles) [101]. These difficult markers might be among the main functional variants contributing to phenotypes.

\section{Beyond one gene-one phenotype: the importance of regulatory contributions}

Almost all the diseases mentioned above are polygenic phenotypes. Among the familial mutations, DTDS clusters carry severely impaired DA reuptake function; others carry 34\% or more impaired function in cases of mental illnesses, likely involving other mechanisms such as efflux or co-existence with other genetic variations. Direct and indirect hDAT networks might be operating for the diverse, "V $V_{\max }$-noncompliant" familial phenotypes.

On the other hand, pathway and cell subtype could mediate its regulatory genetics. Noticeably, $3^{\prime}$ VNTR is related to seven phenotypes (Fig. 2b), with three possible explanations. First, a pathway targeting this locus is hub-connected with others playing critical roles among the seven phenotypes. Consistently, genetic pathways are increasingly recognized in etiology of diseases $[126,127]$. Second, 3'VNTR plays different roles in different celltypes (e.g., caudate vs other striatal regions) [99] since DA neurons are heterogeneous and regulate different elements of brain function [128-130]. Third, some signals such for PTSD and depression arise from LD with their underlying loci. 3'VNTR is the most used marker and accounted for most signals here.

Overall, SLC6A3's multiphenotypic findings can be explained not only by conditional hDAT activity but also by coordination with other genes and the impact of environment and epigenomics during certain sensitive periods in development [131, 132]. In other words, mutations in different parts of SLC6A3 may affect multidimensional dynamics of the protein's activity; or, a phenotype may stand out only when a SLC6A3 functional variant co-exists functionally with other particular variation in the 
1042

subject's genome through pathway action. Those explanations warrant future study using a systems approach for mechanistic elucidation.

\section{Gene-wide functionality}

We have shown that two different haplotypes of the $18 \mathrm{~kb} S L C 6 A 3$ promoter may carry different gene functions and display different responses to the same drug regulators [100]. Based on the critical role of DA transmission, DA transporter haplotype and functional diversity could guide natural selection of genetic variants [133]. We thus examined the $18 \mathrm{~kb}$ promoter regions vs the entire $70 \mathrm{~kb}$ gene, for directed haplotype diversity among $261 \mathrm{KGP}$ populations.

The DNA sequence-based relatedness revealed $>15$ clades for $S L C 6 A 3$, comparing to one major and one minor for the $18 \mathrm{~kb}$ promoter (Fig. 4a vs b). A key feature was that the haplotype diversity was mostly independent of ethnicity but few of the selections were indeed ethnic oriented, consistent with the ADHD findings. For example, Southern Asians or Africans each had at least two distinct selections. By contrast, Europeans and Admixed Americans lacked such selections. Since this approach may reveal functional variation [134], the result suggests that SLC6A3 carry great functional diversity. Given the realization that associations

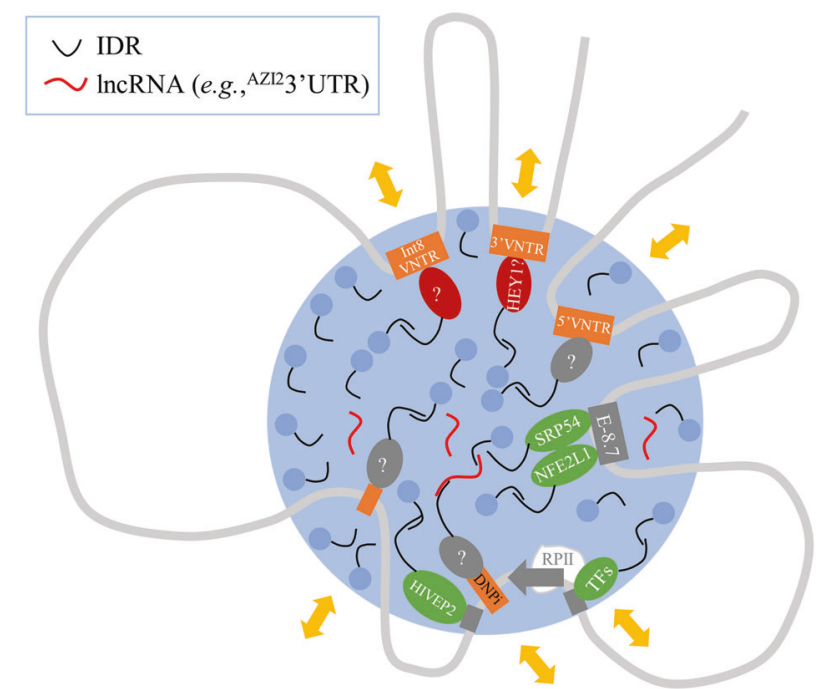

Fig. 5 Multidisease hypothesis for variant- and pathway-related condensate in SLC6A3 transcription. Gray curve, chromosome DNA harboring SLC6A3; orange rectangle, polymorphic sites (unlabeled for Intron 4); gray rectangle, cis-acting element; red oval, repressor; green oval, activator; gray oval, unknown TF as indicated by "?"; light blue dot, other condensation proteins such as Mediator; large blue circle, dynamic condensate; gray arrow, transcription start with RNA polymerase II (RPII) complex; TFs, other known transcription factors such as NURR1, GMEB1, PITX3, FOXA2, LMX1A and SP1/3. Yellow double-arrows, different disease-related and TF-mediated pathways. HEY1 has not been shown with allelic binding yet; majority of the proteins each have multiple IDRs so that this schematic has neither molecular nor dimensional accuracy. can be ethnicity dependent for common diseases including depressive disorder and PD among others [83, 135-138], metaanalyses need to consider ethnic stratification for mechanistic clarification [138-140].

Consistent with the gene-wide selection results, significant epistatic associations with PD vs SUDs pointed to disease-oriented genetic coordination among different functional variants located from the $3^{\prime}$ to the $5^{\prime}$ ends (Fig. 4c). Together, these data suggest that functional variations are located in both promoter and nonpromoter regions, which supports the association signals for diseases in Fig. 2b (throughout the gene) and for protein expression in Fig. 3 (promoter regions).

\section{Gaps in knowledge}

Many pieces are missing in terms of genetic contributions considering pros and cons of LD-based and synthetic association [141], coordination with other genes [102, 142], concurrent epigenetics, cell type-dependence and perhaps polymorphic RNA properties, which have not been examined for this gene. Among them, three key gaps emerge given the current status of our understanding.

Gap one is molecular engagement: multiple cis-acting loci need to be typed at the same time, that is, a multimarker approach considering the extensive genetic recombination (Fig. 2b). This way one could identify a locus-selective association and sort out phenotype-specific pathways. Recent developments in transcription mechanisms uncover the formation of liquid-phased condensate for gene transcription and this condensate may contain various proteins with IDRs and RNAs [143, 144]. Multivalent and weak interactions among the IDRs and RNA contribute to the phase formation. Consistently, known TFs of SLC6A3 may contain IDRs (Supplementary Table 2), along with IncRNA [102], and are well positioned to contribute to such a condensate. This SLC6A3 condensate, which engages both enhancers (such as E-8.7) and silencers (such as $3^{\prime}$ and Intron 8 VNTRs), may enable an initial understanding of a multisite mechanism (Fig. 5).

Gap two is causality. Most clinical investigations generate correlational information. To clarify the genetic contribution, allelic engineering studies in rodents or higher species are needed to examine the behavioral consequences. This approach is now possible with the new genomic editing technologies [145, 146].

Gap three is environmental genetics, an important role in the systems etiology. To fill this gap requires the use of genetically engineered animal models to evaluate the effects of relevant environmental factors.

\section{Conclusion and hypothesis}

SLC6A3 contributes to a spectrum of central nervous diseases and comorbidity in two main ways (Fig. 6). Nonsynonymous mutations affect hDAT's properties; synonymous variations each confer unique spatiotemporal features of the transcription, responses to internal or environmental factors or post-transcriptional RNA properties.

How does SLC6A3 exert its risks? More information is missing than has been accumulated about this question, reminiscent of the parable "blind men and an elephant". An emerging hypothesis

\section{DTDS ASD ADHD BP SUDs/Sleep/SCZ/MDD/PTSD PD/LID}

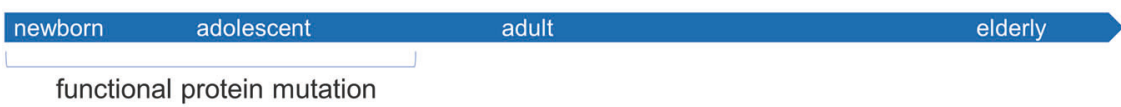

regulatory nucleotide variation

Fig. 6 SLC6A3 genetics $x$ age-dependent expression of phenotypes. Horizontal blue bar, lifespan from newborn at left to elderly at right; associated phenotypes are indicated on the top where red, for psychiatric disorders and black, for movement disorders at the two vulnerable ends of lifespan; associated genetic effects are indicated below the bar; $x$, interaction. 
is pathway-differential condensate (Fig. 5). By this hypothesis, several pathways may regulate $S \angle C 6 A 3$ condensate via TFs partly at polymorphic loci and those same pathways also intersect with other genes so that each pathway connects a set of different genes related with other diseases for shared genetic risks [147]. To test this locus-pathway-phenotype (LPP) hypothesis, we postulate that it requires consideration of multiple functional markers in the same association or functional study and stratification of ethnicities and genders [43].

\section{DATA AVAILABILITY}

Data collected or analyzed by this study are included in this published article (and its Supplementary Files).

\section{REFERENCES}

1. Juarez EJ, Samanez-Larkin GR. Exercise, dopamine, and cognition in older age. Trends Cogn Sci. 2019;23:986-8.

2. Coddington LT, Dudman JT. Learning from action: reconsidering movement signaling in midbrain dopamine neuron activity. Neuron. 2019;104:63-77.

3. Bamford NS, Wightman RM, Sulzer D. Dopamine's effects on corticostriatal synapses during reward-based behaviors. Neuron. 2018;97:494-510.

4. Burke CJ, Tobler PN. Time, not size, matters for striatal reward predictions to dopamine. Neuron. 2016;91:8-11.

5. Satterfield BC, Wisor JP, Schmidt MA, Van Dongen HPA. Time-on-task effect during sleep deprivation in healthy young adults is modulated by dopamine transporter genotype. Sleep. 2017;40:zsx167.

6. Bauckneht M, Chincarini A, De Carli F, Terzaghi M, Morbelli S, Nobili F, et al. Presynaptic dopaminergic neuroimaging in REM sleep behavior disorder: a systematic review and meta-analysis. Sleep Med Rev. 2018;41:266-74.

7. Meng F, Guo Z, Hu Y, Mai W, Zhang Z, Zhang B, et al. CD73-derived adenosine controls inflammation and neurodegeneration by modulating dopamine signalling. Brain: a J Neurol. 2019;142:700-18.

8. Wu Y, Hu Y, Wang B, Li S, Ma C, Liu X, et al. Dopamine uses the DRD5-ARRB2PP2A signaling axis to block the TRAF6-mediated NF-KB pathway and suppress systemic inflammation. Mol Cell. 2020;78:42-56.e46.

9. Gaweda G, lyer RP, Shaver PR, Grilo GA, Dinkins ML, Stoffel HJ, et al. Dopamine receptor D3 agonist (Pramipexole) reduces morphine-induced cardiac fibrosis. Biochemical biophysical Res Commun. 2020;529:1080-5.

10. Wan SH, Stevens SR, Borlaug BA, Anstrom KJ, Deswal A, Felker GM, et al. Differential response to low-dose dopamine or low-dose nesiritide in acute heart failure with reduced or preserved ejection fraction: results from the ROSE AHF Trial (renal optimization strategies evaluation in acute heart failure). Circ Heart Fail. 2016;9:e002593.

11. Wang PS, Walker AM, Tsuang MT, Orav EJ, Glynn RJ, Levin R, et al. Dopamine antagonists and the development of breast cancer. Arch Gen Psychiatry. 2002:59:1147-54.

12. Weissenrieder JS, Neighbors JD, Mailman RB, Hohl RJ. Cancer and the dopamine $\mathrm{D}(2)$ receptor: a pharmacological perspective. J Pharmacol Exp Therapeutics. 2019;370:111-26.

13. Sachlos E, Risueño RM, Laronde S, Shapovalova Z, Lee JH, Russell J, et al. Identification of drugs including a dopamine receptor antagonist that selectively target cancer stem cells. Cell. 2012;149:1284-97.

14. Miller D, Guenther DT, Maurer AP, Hansen CA, Zalesky A, Khoshbouei H. Dopamine transporter is a master regulator of dopaminergic neural network connectivity. J Neurosci: the official journal of the Society for Neuroscience 2021:1:5453-70.

15. Salatino-Oliveira A, Rohde LA, Hutz MH. The dopamine transporter role in psychiatric phenotypes. Am J Med Genet Part B, Neuropsychiatr Genet: Off Publ Int Soc Psychiatr Genet. 2018;177:211-31.

16. Fang $Y$, Ronnekleiv OK. Cocaine upregulates the dopamine transporter in fetal rhesus monkey brain. J Neurosci: Off J Soc Neurosci. 1999;19:8966-78.

17. Letchworth SR, Sexton T, Childers SR, Vrana KE, Vaughan RA, Davies HM, et al. Regulation of rat dopamine transporter mRNA and protein by chronic cocaine administration. J Neurochemistry. 1999;73:1982-9.

18. Letchworth SR, Daunais JB, Hedgecock AA, Porrino LJ. Effects of chronic cocaine administration on dopamine transporter mRNA and protein in the rat. Brain Res. 1997;750:214-22.

19. Filipenko ML, Alekseyenko OV, Beilina AG, Kamynina TP, Kudryavtseva NN. Increase of tyrosine hydroxylase and dopamine transporter mRNA levels in ventral tegmental area of male mice under influence of repeated aggression experience. Brain Res Mol Brain Res. 2001;96:77-81.
20. Redina O, Babenko V, Smagin D, Kovalenko I, Galyamina A, Efimov V, et al. Gene exprbession changes in the ventral tegmental area of male mice with alternative social behavior experience in chronic agonistic interactions. Int J Mol Sci. 2020;21:6599.

21. Ong ZY, Muhlhausler BS. Maternal "junk-food" feeding of rat dams alters food choices and development of the mesolimbic reward pathway in the offspring. FASEB J: Off Publ Federation Am Societies Exp Biol. 2011;25:2167-79.

22. Yuan ZY, Gu P, Liu L, Wang YY, Liu J, Cui DS, et al. Neuroprotective effects of enriched environment in MPTP-treated SAMP8 mice. Neurosci Lett. 2009;454:6-10.

23. Amato D, Canneva F, Cumming P, Maschauer S, Groos D, Dahlmanns JK, et al. A dopaminergic mechanism of antipsychotic drug efficacy, failure, and failure reversal: the role of the dopamine transporter. Mol Psychiatr. 2020;25:2101-18.

24. Sheng $Y$, Filichia $E$, Shick E, Preston KL, Phillips KA, Cooperman L, et al. Using iPSC-derived human DA neurons from opioid-dependent subjects to study dopamine dynamics. Brain Behav. 2016;6:e00491.

25. Figlewicz DP, Szot P, Chavez M, Woods SC, Veith RC. Intraventricular insulin increases dopamine transporter mRNA in rat VTA/substantia nigra. Brain Res. 1994;644:331-4.

26. Wiers CE, Lohoff FW, Lee J, Muench C, Freeman C, Zehra A, et al. Methylation of the dopamine transporter gene in blood is associated with striatal dopamine transporter availability in ADHD: a preliminary study. Eur J Neurosci. 2018;48:1884-95.

27. Shumay E, Fowler JS, Volkow ND. Genomic features of the human dopamine transporter gene and its potential epigenetic States: implications for phenotypic diversity. PloS ONE. 2010;5:e11067.

28. Hochstatter KR, Hull SJ, Sethi AK, Burns ME, Mundt MP, Westergaard RP. Promoting safe injection practices, substance use reduction, hepatitis $C$ testing, and overdose prevention among syringe service program clients using a computertailored intervention: pilot randomized controlled trial. J Med Internet Res. 2020;22:e19703.

29. Westergaard RP, Hull SJ, Merkow A, Stephens LK, Hochstatter KR, Olson-Streed $\mathrm{HK}$, et al. Computerized tailored interventions to enhance prevention and screening for hepatitis $C$ virus among people who inject drugs: protocol for a randomized pilot study. JMIR Res Protoc. 2016;5:e15.

30. Xiong N, Schiller MR, Li J, Chen X, Lin Z. Severe COVID-19 in Alzheimer's disease: APOE4's fault again? Alzheimer's Res Ther. 2021;13:111.

31. Wang KH, Penmatsa A, Gouaux E. Neurotransmitter and psychostimulant recognition by the dopamine transporter. Nature. 2015;521:322-7.

32. Aggarwal S, Liu X, Rice C, Menell P, Clark PJ, Paparoidamis N, et al. Identification of a novel allosteric modulator of the human dopamine transporter. ACS Chem Neurosci. 2019;10:3718-30.

33. Sudmant PH, Rausch T, Gardner EJ, Handsaker RE, Abyzov A, Huddleston J, et al. An integrated map of structural variation in 2504 human genomes. Nature. 2015;526:75-81.

34. Gao F, Ming C, Hu W, Li H. New software for the fast estimation of population recombination rates (FastEPRR) in the Genomic Era. G3 (Bethesda, Md). 2016;6:1563-71.

35. Wang GJ, Volkow ND, Wigal T, Kollins SH, Newcorn JH, Telang F, et al. Long-term stimulant treatment affects brain dopamine transporter level in patients with attention deficit hyperactive disorder. PloS ONE. 2013;8:e63023.

36. Volkow ND, Wang GJ, Fowler JS, Logan J, Franceschi D, Maynard L, et al. Relationship between blockade of dopamine transporters by oral methylphenidate and the increases in extracellular dopamine: therapeutic implications. Synapse. 2002;43:181-7.

37. Fowler JS, Volkow ND, Wang GJ, Gatley SJ, Logan J. [(11)]Cocaine: PET studies of cocaine pharmacokinetics, dopamine transporter availability and dopamine transporter occupancy. Nucl Med Biol. 2001;28:561-72.

38. Zhou Y, Michelhaugh SK, Schmidt CJ, Liu JS, Bannon MJ, Lin Z. Ventral midbrain correlation between genetic variation and expression of the dopamine transporter gene in cocaine-abusing versus non-abusing subjects. Addiction Biol. 2014;19:122-31.

39. Li Z, Zhang Z, He Z, Tang W, Li T, Zeng Z. et al. A partition-ligation-combinationsubdivision EM algorithm for haplotype inference with multiallelic markers: update of the SHEsis. Cell Res. 2009;19:519-23. http://analysis.bio-x.cn.

40. Kennedy JL, Xiong N, Yu J, Zai CC, Pouget JG, Li J, et al. Increased Nigral SLC6A3 activity in schizophrenia patients: findings from the toronto-McLean Cohorts. Schizophr Bull. 2016;42:772-81.

41. Thompson JD, Gibson TJ, Higgins DG. Multiple sequence alignment using ClustalW and ClustalX. Current protocols in bioinformatics 2002; Chapter 2: Unit 2.3.

42. Page RD. TreeView: an application to display phylogenetic trees on personal computers. Computer Appl Biosci: CABIOS. 1996;12:357-8. 
43. Liu K, Zhao J, Chen C, Xu J, Bell RL, Hall FS, et al. Epistatic evidence for genderdependant slow neurotransmission signalling in substance use disorders: PPP1R12B versus PPP1R1B. EBioMedicine. 2020;61:103066.

44. Necci M, Piovesan D, Clementel D, Dosztányi Z, Tosatto SCE. MobiDB-lite 3.0: fast consensus annotation of intrinsic disorder flavours in proteins. Bioinformatics (Oxford, England) 2020

45. Kurian MA. SLC6A3-Related Dopamine Transporter Deficiency Syndrome. In: Adam MP, Ardinger HH, Pagon RA, Wallace SE, Bean LJH, Stephens K, et al. (eds). GeneReviews( ${ }^{\oplus}$ ). University of Washington, Seattle Copyright ${ }^{\odot}$ 1993-2020, University of Washington, Seattle. GeneReviews is a registered trademark of the University of Washington, Seattle. All rights reserved.: Seattle (WA), 1993.

46. Kurian MA, Li Y, Zhen J, Meyer E, Hai N, Christen HJ, et al. Clinical and molecular characterisation of hereditary dopamine transporter deficiency syndrome: an observational cohort and experimental study. Lancet Neurol. 2011;10:54-62.

47. Kurian MA, Zhen J, Cheng SY, Li Y, Mordekar SR, Jardine P, et al. Homozygous lossof-function mutations in the gene encoding the dopamine transporter are associated with infantile parkinsonism-dystonia. J Clin Investig. 2009;119:1595-603.

48. $\mathrm{Ng} \mathrm{J}$, Zhen J, Meyer E, Erreger K, Li Y, Kakar N, et al. Dopamine transporter deficiency syndrome: phenotypic spectrum from infancy to adulthood. Brain: J Neurol. 2014;137:1107-19.

49. Baga M, Spagnoli C, Soliani L, Salerno GG, Rizzi S, Frattini D, et al. Early-onset dopamine transporter deficiency syndrome: long-term follow-up. Can J Neurol Sci Le journal canadien des sciences neurologiques 2021;48:285-6.

50. Heidari E, Razmara E, Hosseinpour S, Tavasoli AR, Garshasbi M. Homozygous inframe variant of SCL6A3 causes dopamine transporter deficiency syndrome in a consanguineous family. Ann Hum Genet. 2020;84:315-23.

51. Nasehi MM, Nikkhah A, Salari M, Soltani P, Shirzadi S. Dopamine transporter deficiency syndrome: a case with hyper- and hypokinetic extremes. Mov Disord Clin Pract. 2020;7:S57-60.

52. Campbell NG, Shekar A, Aguilar JI, Peng D, Navratna V, Yang D, et al. Structural, functional, and behavioral insights of dopamine dysfunction revealed by a deletion in SLC6A3. Proc Natl Acad Sci USA. 2019;116:3853-62.

53. Reith MEA, Jones KT, Zhen J, Topiol S. Latch and trigger role for R445 in DAT transport explains molecular basis of DTDS. Bioorg Medicinal Chem Lett. 2018;28:470-5.

54. Hansen FH, Skjørringe T, Yasmeen S, Arends NV, Sahai MA, Erreger K, et al. Missense dopamine transporter mutations associate with adult parkinsonism and ADHD. The. J Clin Investig. 2014;124:3107-20.

55. Herborg F, Andreassen TF, Berlin F, Loland CJ, Gether U. Neuropsychiatric disease-associated genetic variants of the dopamine transporter display heterogeneous molecular phenotypes. J Biol Chem. 2018;293:7250-62.

56. DiCarlo GE, Aguilar JI, Matthies HJ, Harrison FE, Bundschuh KE, West $A$, et al. Autism-linked dopamine transporter mutation alters striatal dopamine neurotransmission and dopamine-dependent behaviors. J Clin Investig. 2019;129:3407-19.

57. Hamilton PJ, Campbell NG, Sharma S, Erreger K, Herborg Hansen F, Saunders C, et al. De novo mutation in the dopamine transporter gene associates dopamine dysfunction with autism spectrum disorder. Mol Psychiatry. 2013;18:1315-23.

58. Sakrikar D, Mazei-Robison MS, Mergy MA, Richtand NW, Han Q, Hamilton PJ, et al. Attention deficit/hyperactivity disorder-derived coding variation in the dopamine transporter disrupts microdomain targeting and trafficking regulation. J Neurosci: Off J Soc Neurosci. 2012;32:5385-97.

59. Herborg F, Jensen KL, Tolstoy S, Arends NV, Posselt LP, Shekar A, et al. Identifying dominant-negative actions of a dopamine transporter variant in patients with parkinsonism and neuropsychiatric disease. JCI Insight. 2021;16:e151496.

60. Bowton E, Saunders C, Reddy IA, Campbell NG, Hamilton PJ, Henry LK, et al. SLC6A3 coding variant Ala559Val found in two autism probands alters dopamine transporter function and trafficking. Transl Psychiatry. 2014;4:e464.

61. Mazei-Robison MS, Bowton E, Holy M, Schmudermaier M, Freissmuth $M$, Sitte $\mathrm{HH}$, et al. Anomalous dopamine release associated with a human dopamine transporter coding variant. J Neurosci: Off J Soc Neurosci. 2008;28:7040-6.

62. Horschitz S, Hummerich R, Lau T, Rietschel M, Schloss P. A dopamine transporter mutation associated with bipolar affective disorder causes inhibition of transporter cell surface expression. Mol Psychiatry. 2005;10:1104-9.

63. Cartier E, Hamilton PJ, Belovich AN, Shekar A, Campbell NG, Saunders C, et al. Rare autism-associated variants implicate syntaxin 1 (STX1 R26Q) phosphorylation and the dopamine transporter (hDAT R51W) in dopamine neurotransmission and behaviors. EBioMedicine. 2015;2:135-46.

64. Grünhage F, Schulze TG, Müller DJ, Lanczik M, Franzek E, Albus M, et al. Systematic screening for DNA sequence variation in the coding region of the human dopamine transporter gene (DAT1). Mol Psychiatry. 2000;5:275-82.

65. Kovtun O, Sakrikar D, Tomlinson ID, Chang JC, Arzeta-Ferrer X, Blakely RD, et al. Single-quantum-dot tracking reveals altered membrane dynamics of an attention-deficit/hyperactivity-disorder-derived dopamine transporter coding variant. ACS Chem Neurosci. 2015;6:526-34.
66. Rojas G, Orellana I, Rosales-Rojas R, García-Olivares J, Comer J, Vergara-Jaque A. Structural determinants of the dopamine transporter regulation mediated by $G$ proteins. J Chem Inf Modeling. 2020;60:3577-86.

67. Cervinski MA, Foster JD, Vaughan RA. Syntaxin 1A regulates dopamine transporter activity, phosphorylation and surface expression. Neuroscience. 2010;170:408-16.

68. Biological insights from 108 schizophrenia-associated genetic loci. Nature 2014;41:5453-70

69. Demontis D, Walters RK, Martin J, Mattheisen M, Als TD, Agerbo E, et al. Discovery of the first genome-wide significant risk loci for attention deficit/ hyperactivity disorder. Nat Genet. 2019;51:63-75.

70. Sanchez-Roige S, Palmer AA, Fontanillas P, Elson SL, Adams MJ, Howard DM, et al. Genome-wide association study meta-analysis of the alcohol use disorders identification test (AUDIT) in two population-based cohorts. Am J Psychiatry. 2019;176:107-18.

71. Ma Y, Fan R, Li MD. Meta-analysis reveals significant association of the 3'-UTR VNTR in SLC6A3 with alcohol dependence. Alcohol Clin Exp Res. 2016;40:1443-53.

72. Kibitov AO, Ivashchenko DV, Brodyansky VM, Chuprova NA, Shuvalov SA. [Combination of DAT and DBH gene polymorphisms with a family history of alcohol use disorders increases the risk of withdrawal seizures and delirium tremens during alcohol withdrawal in alcohol-dependent men]. Zhurnal nevrologii i psikhiatrii imeni SS Korsakova. 2016;116:68-80.

73. Schacht JP, Anton RF, McNamara PJ, Im Y, King AC. The dopamine transporter VNTR polymorphism moderates the relationship between acute response to alcohol and future alcohol use disorder symptoms. Addiction Biol. 2019;24:1109-18.

74. Ma Y, Yuan W, Cui W, Li MD. Meta-analysis reveals significant association of $3^{\prime}$ UTR VNTR in SLC6A3 with smoking cessation in Caucasian populations. Pharmacogenomics J. 2016;16:10-17.

75. Li S, Wang Q, Pan L, Li H, Yang X, Jiang F, et al. The association of dopamine pathway gene score, nicotine dependence and smoking cessation in a rural male population of Shandong, China. Am J Addictions. 2016;25:493-8.

76. Tiili EM, Mitiushkina NV, Sukhovskaya OA, Imyanitov EN, Hirvonen AP. The effect of SLC6A3 variable number of tandem repeats and methylation levels on individual susceptibility to start tobacco smoking and on the ability of smokers to quit smoking. Pharmacogenetics Genomics. 2020;30:117-23.

77. Grünblatt E, Werling AM, Roth A, Romanos M, Walitza S. Association study and a systematic meta-analysis of the VNTR polymorphism in the 3'-UTR of dopamine transporter gene and attention-deficit hyperactivity disorder. J Neural Transm (Vienna, Austria: 1996). 2019;126:517-29.

78. Li L, Bao Y, He S, Wang G, Guan Y, Ma D, et al. The Association between genetic variants in the dopaminergic system and posttraumatic stress disorder: a metaanalysis. Medicine. 2016:95:e3074.

79. Bieliński $M$, Jaracz $M$, Lesiewska $N$, Tomaszewska $M$, Sikora $M$, Junik $R$, et al. Association between COMT Val158Met and DAT1 polymorphisms and depressive symptoms in the obese population. Neuropsychiatr Dis Treat. 2017;13:2221-9.

80. López-León S, Janssens AC, González-Zuloeta Ladd AM, Del-Favero J, Claes SJ, Oostra BA, et al. Meta-analyses of genetic studies on major depressive disorder. Mol Psychiatry. 2008;13:772-85.

81. Xu FL, Ding M, Wu X, Liu YP, Xia X, Yao J, et al. A Meta-analysis of the Association between SLC6A3 gene polymorphisms and schizophrenia. J Mol Neurosci: MN. 2020;70:155-66.

82. Rhodes JA, Lane JM, Vlasac IM, Rutter MK, Czeisler CA, Saxena R. Association of DAT1 genetic variants with habitual sleep duration in the UK Biobank. Sleep. 2019;42:zsy193.

83. Zhai D, Li S, Zhao Y, Lin Z. SLC6A3 is a risk factor for Parkinson's disease: a metaanalysis of sixteen years' studies. Neurosci Lett. 2014;564:99-104.

84. van Munster BC, Yazdanpanah M, Tanck MW, de Rooij SE, van de Giessen E, Sijbrands EJ, et al. Genetic polymorphisms in the DRD2, DRD3, and SLC6A3 gene in elderly patients with delirium. Am J Med Genet Part B, Neuropsychiatr Genet: Off Publ Int Soc Psychiatr Genet. 2010;153b:38-45.

85. van Munster BC, de Rooij S, Yazdanpanah M, Tienari PJ, Pitkälä KH, Osse RJ, et al. The association of the dopamine transporter gene and the dopamine receptor 2 gene with delirium, a meta-analysis. Am J Med Genet Part B, Neuropsychiatr Genet: Off Publ Int Soc Psychiatr Genet. 2010;153b:648-55.

86. Pinsonneault JK, Han DD, Burdick KE, Kataki M, Bertolino A, Malhotra AK, et al. Dopamine transporter gene variant affecting expression in human brain is associated with bipolar disorder. Neuropsychopharmacol: Off Publ Am Coll Neuropsychopharmacol. 2011;36:1644-55.

87. Myer NM, Boland JR, Faraone SV. Pharmacogenetics predictors of methylphenidate efficacy in childhood ADHD. Mol Psychiatry. 2018;23:1929-36.

88. Guin D, Mishra MK, Talwar P, Rawat C, Kushwaha SS, Kukreti S, et al. A systematic review and integrative approach to decode the common molecular link 
between levodopa response and Parkinson's disease. BMC Med Genomics. 2017;10:56.

89. Politi C, Ciccacci C, Novelli G, Borgiani P. Genetics and treatment response in Parkinson's disease: an update on pharmacogenetic studies. Neuromolecular Med. 2018;20:1-17.

90. Kaplan N, Vituri A, Korczyn AD, Cohen OS, Inzelberg R, Yahalom G, et al. Sequence variants in SLC6A3, DRD2, and BDNF genes and time to levodopainduced dyskinesias in Parkinson's disease. J Mol Neurosci: MN. 2014;53:183-8.

91. Purcaro C, Vanacore N, Moret F, Di Battista ME, Rubino A, Pierandrei S, et al. DAT gene polymorphisms (rs28363170, rs393795) and levodopa-induced dyskinesias in Parkinson's disease. Neurosci Lett. 2019;690:83-8.

92. Redenšek S, Flisar D, Kojović M, Gregorič Kramberger M, Georgiev D, Pirtošek Z, et al. Dopaminergic pathway genes influence adverse events related to dopaminergic treatment in Parkinson's Disease. Front Pharmacol. 2019;10:8.

93. Fuke S, Suo S, Takahashi N, Koike H, Sasagawa N, Ishiura S. The VNTR polymorphism of the human dopamine transporter (DAT1) gene affects gene expression. Pharmacogenomics J. 2001;1:152-6.

94. Mill J, Asherson P, Craig I, D'Souza UM. Transient expression analysis of allelic variants of a VNTR in the dopamine transporter gene (DAT1). BMC Genet. 2005;6:3.

95. VanNess SH, Owens MJ, Kilts CD. The variable number of tandem repeats element in DAT1 regulates in vitro dopamine transporter density. BMC Genet. 2005;6:55

96. Faraone SV, Spencer TJ, Madras BK, Zhang-James Y, Biederman J. Functional effects of dopamine transporter gene genotypes on in vivo dopamine transporter functioning: a meta-analysis. Mol Psychiatry. 2014;19:880-9.

97. Hill M, Anney RJ, Gill M, Hawi Z. Functional analysis of intron 8 and $3^{\prime}$ UTR variable number of tandem repeats of SLC6A3: differential activity of intron 8 variants. Pharmacogenomics J. 2010;10:442-7.

98. Guindalini C, Howard M, Haddley K, Laranjeira R, Collier D, Ammar N, et al. A dopamine transporter gene functional variant associated with cocaine abuse in a Brazilian sample. Proc Natl Acad Sci USA. 2006;103:4552-7.

99. Shumay E, Chen J, Fowler JS, Volkow ND. Genotype and ancestry modulate brain's DAT availability in healthy humans. PloS ONE. 2011;6:e22754.

100. Zhao Y, Xiong N, Liu Y, Zhou Y, Li N, Qing H, et al. Human dopamine transporter gene: differential regulation of $18-\mathrm{kb}$ haplotypes. Pharmacogenomics. 2013;14:1481-94.

101. Zhao J, Zhou $Y$, Xiong N, Qing H, Wang T, Lin Z. Presence of recombination hotspots throughout SLC6A3. PloS ONE. 2019;14:e0218129.

102. Liu K, Yu J, Zhao J, Zhou Y, Xiong N, Xu J, et al. (AZI2)3'UTR Is a New SLC6A3 downregulator associated with an epistatic protection against substance use disorders. Mol Neurobiol. 2018;55:5611-22.

103. Savelieva KV, Caudle WM, Findlay GS, Caron MG, Miller GW. Decreased ethanol preference and consumption in dopamine transporter female knock-out mice. Alcohol Clin Exp Res. 2002;26:758-64.

104. Song R, Zhang HY, Li X, Bi GH, Gardner EL, Xi ZX. Increased vulnerability to cocaine in mice lacking dopamine D3 receptors. Proc Natl Acad Sci U.S.A. 2012;109:17675-80.

105. Liu K, Yu J, Zhao J, Zhou Y, Xiong N, Xu J, et al. (AZI2)3'UTR Is a New SLC6A3 downregulator associated with an epistatic protection against substance use disorders. Mol Neurobiol. 2018;55;5611-22.

106. Liu QR, Canseco-Alba A, Zhang HY, Tagliaferro P, Chung M, Dennis E, et al. Cannabinoid type 2 receptors in dopamine neurons inhibits psychomotor behaviors, alters anxiety, depression and alcohol preference. Sci Rep. 2017;7:17410.

107. Diana M. The dopamine hypothesis of drug addiction and its potential therapeutic value. Front Psychiatry. 2011;2:64.

108. Martinez D, Greene K, Broft A, Kumar D, Liu F, Narendran R, et al. Lower level of endogenous dopamine in patients with cocaine dependence: findings from PET imaging of $\mathrm{D}(2) / \mathrm{D}(3)$ receptors following acute dopamine depletion. Am J Psychiatry. 2009;166:1170-7.

109. Pickens CL, Calu DJ. Alcohol reward, dopamine depletion, and GDNF. J Neurosci. 2011;31:14833-4.

110. Dackis CA, Gold MS. New concepts in cocaine addiction: the dopamine depletion hypothesis. Neurosci Biobehav Rev. 1985;9:469-77.

111. Copeland BJ, Neff NH, Hadjiconstantinou M. Enhanced dopamine uptake in the striatum following repeated restraint stress. Synapse. 2005;57:167-74.

112. Li S, Kim KY, Kim JH, Kim JH, Park MS, Bahk JY, et al. Chronic nicotine and smoking treatment increases dopamine transporter mRNA expression in the rat midbrain. Neurosci Lett. 2004;363:29-32.

113. Hadjiconstantinou M, Duchemin AM, Zhang H, Neff NH. Enhanced dopamine transporter function in striatum during nicotine withdrawal. Synap (N. Y, NY). 2011;65:91-8.

114. Taylor M, Collin SM, Munafò MR, MacLeod J, Hickman M, Heron J. Patterns of cannabis use during adolescence and their association with harmful substance use behaviour: findings from a UK birth cohort. J Epidemiol Community Health. 2017;71:764-70.

115. Thrul J, Rabinowitz JA, Reboussin BA, Maher BS, lalongo NS. Adolescent cannabis and tobacco use are associated with opioid use in young adulthood12-year longitudinal study in an urban cohort. Addiction (Abingdon, England) 2020.

116. Williams AR. Cannabis as a gateway drug for opioid use disorder. J Law Med Ethics: a J Am Soc Law Med Ethics. 2020;48:268-74.

117. Pistis M, Perra S, Pillolla G, Melis M, Muntoni AL, Gessa GL. Adolescent exposure to cannabinoids induces long-lasting changes in the response to drugs of abuse of rat midbrain dopamine neurons. Biol Psychiatry. 2004;56:86-94.

118. Zhang HY, Gao M, Liu QR, Bi GH, Li X, Yang HJ, et al. Cannabinoid CB2 receptors modulate midbrain dopamine neuronal activity and dopamine-related behavior in mice. Proc Natl Acad Sci USA. 2014;111:E5007-15.

119. Canseco-Alba A, Schanz N, Sanabria B, Zhao J, Lin Z, Liu QR, et al. Behavioral effects of psychostimulants in mutant mice with cell-type specific deletion of CB2 cannabinoid receptors in dopamine neurons. Behavioural Brain Res. 2019;360:286-97.

120. Pizzagalli DA, Berretta S, Wooten D, Goer F, Pilobello KT, Kumar P, et al. Assessment of striatal dopamine transporter binding in individuals with major depressive disorder: in vivo positron emission tomography and postmortem evidence. JAMA Psychiatry. 2019;76:854-61.

121. Bahi A, Dreyer JL. Dopamine transporter (DAT) knockdown in the nucleus accumbens improves anxiety- and depression-related behaviors in adult mice. Behavioural Brain Res. 2019;359:104-15.

122. Perona MT, Waters S, Hall FS, Sora I, Lesch KP, Murphy DL, et al. Animal models of depression in dopamine, serotonin, and norepinephrine transporter knockout mice: prominent effects of dopamine transporter deletions. Behavioural Pharmacol. 2008;19:566-74.

123. Zhao Y, Yu J, Zhao J, Chen X, Xiong N, Wang T, et al. Intragenic transcriptional cis-Antagonism across SLC6A3. Mol Neurobiol. 2019;56:4051-60.

124. Guarnerio J, Zhang Y, Cheloni G, Panella R, Mae Katon J, Simpson M, et al. Intragenic antagonistic roles of protein and circRNA in tumorigenesis. Cell Res. 2019;29:628-40.

125. Zeidler $M$, Hüttenhofer $A$, Kress $M$, Kummer KK. Intragenic MicroRNAs autoregulate their host genes in both direct and indirect ways-A cross-species analysis. Cells. 2020;9:232.

126. Siljee JE, Wang Y, Bernard AA, Ersoy BA, Zhang S, Marley A, et al. Subcellular localization of MC4R with $A D C Y 3$ at neuronal primary cilia underlies a common pathway for genetic predisposition to obesity. Nat Genet. 2018;50:180-5.

127. Kibinge NK, Relton CL, Gaunt TR, Richardson TG. Characterizing the causal pathway for genetic variants associated with neurological phenotypes using human brain-derived proteome data. Am J Hum Genet. 2020;106:885-92.

128. Engelhard B, Finkelstein J, Cox J, Fleming W, Jang HJ, Ornelas S, et al. Specialized coding of sensory, motor and cognitive variables in VTA dopamine neurons. Nature. 2019;570:509-13.

129. Matsumoto M, Hikosaka O. Two types of dopamine neuron distinctly convey positive and negative motivational signals. Nature. 2009;459:837-41.

130. Lammel S, Lim BK, Ran C, Huang KW, Betley MJ, Tye KM, et al. Input-specific control of reward and aversion in the ventral tegmental area. Nature. 2012;491:212-7.

131. Luby JL, Baram TZ, Rogers CE, Barch DM. Neurodevelopmental optimization after early-life adversity: cross-species studies to elucidate sensitive periods and brain mechanisms to inform early intervention. Trends Neurosci. 2020;43:744-51.

132. Belbin O, Morgan K, Medway C, Warden D, Cortina-Borja M, van Duijn CM, et al. The epistasis project: a multi-cohort study of the effects of BDNF, DBH, and SORT1 epistasis on alzheimer's disease risk. J Alzheimer's Dis: JAD. 2019;68:1535-47.

133. Lin Z, Zhao Y, Chung CY, Zhou Y, Xiong N, Glatt CE, et al. High regulatability favors genetic selection in SLC18A2, a vesicular monoamine transporter essential for life. FASEB J: Off Publ Federation Am Societies Exp Biol. 2010;24:2191-200.

134. Griesmann M, Chang Y, Liu X, Song Y, Haberer G, Crook MB, et al. Phylogenomics reveals multiple losses of nitrogen-fixing root nodule symbiosis. Science (New York, NY) 2018; 361.

135. Avinun R, Nevo A, Radtke SR, Brigidi BD, Hariri AR. Divergence of an association between depressive symptoms and a dopamine polygenic score in Caucasians and Asians. Eur Arch Psychiatry Clin Neurosci. 2020;270:229-35.

136. Chen MH, Raffield LM, Mousas A, Sakaue S, Huffman JE, Moscati A, et al. Transethnic and ancestry-specific blood-cell genetics in 746,667 individuals from 5 Global Populations. Cell. 2020;182:1198-213.e1114.

137. Stryjecki C, Alyass A, Meyre D. Ethnic and population differences in the genetic predisposition to human obesity. Obes Rev: Off J Int Assoc Study Obes. 2018;19:62-80. 
1046

138. Zavala VA, Bracci PM, Carethers JM, Carvajal-Carmona L, Coggins NB, CruzCorrea MR, et al. Cancer health disparities in racial/ethnic minorities in the United States. Br J Cancer. 2021;124:315-32.

139. Lopez KN, Morris SA, Sexson Tejtel SK, Espaillat A, Salemi JL. US mortality attributable to congenital heart disease across the lifespan from 1999 through 2017 exposes persistent racial/ethnic disparities. Circulation. 2020;142:1132-47.

140. Coram MA, Fang H, Candille SI, Assimes TL, Tang H. Leveraging multi-ethnic evidence for risk assessment of quantitative traits in minority populations. Am J Hum Genet. 2017;101:218-26.

141. Dickson SP, Wang K, Krantz I, Hakonarson H, Goldstein DB. Rare variants create synthetic genome-wide associations. PLoS Biol. 2010;8:e1000294.

142. Talkowski ME, Kirov G, Bamne M, Georgieva L, Torres G, Mansour H, et al. A network of dopaminergic gene variations implicated as risk factors for schizophrenia. Hum Mol Genet. 2008;17:747-58.

143. Sabari BR, Dall'Agnese A, Young RA. Biomolecular condensates in the nucleus. Trends Biochemical Sci. 2020;45:961-77.

144. Statello L, Guo CJ, Chen LL, Huarte M. Gene regulation by long non-coding RNAs and its biological functions. Nat Rev Mol Cell Biol. 2021;22:96-118.

145. Wu J, Tang B, Tang Y. Allele-specific genome targeting in the development of precision medicine. Theranostics. 2020;10:3118-37.

146. Zhao J, Lai L, Ji W, Zhou Q. Genome editing in large animals: current status and future prospects. Natl Sci Rev. 2019;6:402-20.

147. Evangelou E, Gao H, Chu C, Ntritsos G, Blakeley P, Butts AR, et al. New alcoholrelated genes suggest shared genetic mechanisms with neuropsychiatric disorders. Nat Hum Behav. 2019;3:950-61.

\section{ACKNOWLEDGEMENTS}

We thank the US National Institutes of Health $(\mathrm{NIH})$ for granting ZL with access to the dbGaP GWAS datasets, and Yong-Yong Shi and Zhi-Qiang Li of Shanghai Jiao Tong University for assistance in haplotype inference.

\section{AUTHOR CONTRIBUTIONS}

ZL conceived the study and ZL, MEAR, NDV contributed to the design; ZL, SK, CEW, HS, MAK and NDV contributed to data collection and analyses; ZL drafted the paper; ZL, MEAR, SK, CEW, MAK, AG, and VDN revised, and finalized the paper.

\section{FUNDING}

This work was supported by the U.S. National Institute on Drug Abuse grants (NIDA) DA031573 and DA021409 (ZL) and DA019676 (MEAR), National Institute on Alcohol Abuse and Alcoholism grants (NIAAA) AA026663 (ZL), and DA035263 (AG), and Y1AA3009 (NDV). NIDA and NIAAA had no further role in paper preparation, or in the decision to submit the paper for publication.

\section{COMPETING INTERESTS}

The authors declare no competing interests.

\section{ADDITIONAL INFORMATION}

Supplementary information The online version contains supplementary material available at https://doi.org/10.1038/s41380-021-01341-5.

Correspondence and requests for materials should be addressed to Zhicheng Lin.

Reprints and permission information is available at http://www.nature.com/ reprints

Publisher's note Springer Nature remains neutral with regard to jurisdictional claims in published maps and institutional affiliations.

Open Access This article is licensed under a Creative Commons Attribution 4.0 International License, which permits use, sharing, adaptation, distribution and reproduction in any medium or format, as long as you give appropriate credit to the original author(s) and the source, provide a link to the Creative Commons license, and indicate if changes were made. The images or other third party material in this article are included in the article's Creative Commons license, unless indicated otherwise in a credit line to the material. If material is not included in the article's Creative Commons license and your intended use is not permitted by statutory regulation or exceeds the permitted use, you will need to obtain permission directly from the copyright holder. To view a copy of this license, visit http://creativecommons. org/licenses/by/4.0/.

(c) The Author(s) 2021 